ARTICLE

\title{
Complete loss of H3K9 methylation dissolves mouse heterochromatin organization
}

Thomas Montavon (10 1, Nicholas Shukeir ${ }^{1}$, Galina Erikson'1, Bettina Engist', Megumi Onishi-Seebacher ${ }^{1,3}$, Devon Ryan 1,4, Yaarub Musa1,5, Gerhard Mittler ${ }^{1}$, Alexandra Graff Meyer², Christel Genoud ${ }^{2}$ \& Thomas Jenuwein (1D) ${ }^{1 凶}$

Histone $\mathrm{H} 3$ lysine 9 ( $\mathrm{H} 3 \mathrm{~K} 9$ ) methylation is a central epigenetic modification that defines heterochromatin from unicellular to multicellular organisms. In mammalian cells, H3K9 methylation can be catalyzed by at least six distinct SET domain enzymes: Suv39h1/Suv39h2, Eset1/Eset2 and G9a/Glp. We used mouse embryonic fibroblasts (MEFs) with a conditional mutation for Eset1 and introduced progressive deletions for the other SET domain genes by CRISPR/Cas9 technology. Compound mutant MEFs for all six SET domain lysine methyltransferase (KMT) genes lack all H3K9 methylation states, derepress nearly all families of repeat elements and display genomic instabilities. Strikingly, the 6KO H3K9 KMT MEF cells no longer maintain heterochromatin organization and have lost electron-dense heterochromatin. This is a compelling analysis of H3K9 methylation-deficient mammalian chromatin and reveals a definitive function for H3K9 methylation in protecting heterochromatin organization and genome integrity.

\footnotetext{
${ }^{1}$ Max-Planck Institute of Immunobiology and Epigenetics, Freiburg, Germany. ${ }^{2}$ Friedrich Miescher Institute for Biomedical Research, Basel, Switzerland. ${ }^{3}$ Present address: Novartis Institute for Biomedical Research (NIBR), Basel, Switzerland. ${ }^{4}$ Present address: Genedata AG, Basel, Switzerland. ${ }^{5}$ Present address: Thermo Fisher Scientific GmbH, Dreieich, Germany. ${ }^{凶}$ email: jenuwein@ie-freiburg.mpg.de
} 
eterochromatin is gene-poor, repeat-rich regions of the genome that are classically defined to remain condensed during interphase ${ }^{1}$. From these early cytological studies, DAPI staining of A/T-rich repeats to high-resolution microscopy, heterochromatin can be described as an electron-dense chromatin material that shows the concentration in subnuclear compartments and is often associated with the nuclear periphery. Heterochromatin has important functions for chromosome segregation, genome integrity, and gene regulation ${ }^{2,3}$.

The methylation of histone $\mathrm{H} 3$ at lysine 9 (H3K9) is a hallmark of heterochromatin. It is established by three distinct enzymatic systems that target different regions of the genome. Suv39h enzymes (Suv39h1 and Suv39h2 in mammals) establish H3K9 trimethylation (H3K9me3) at constitutive heterochromatin ${ }^{4,5}$. Setdb1/Eset1 is required for retrotransposon silencing ${ }^{6,7}$. G9a and Glp are predominantly mediating $\mathrm{H} 3 \mathrm{~K} 9$ dimethylation $(\mathrm{H} 3 \mathrm{~K} 9 \mathrm{me} 2)$ and gene repression at euchromatic loci ${ }^{8,9}$.

A biochemical pathway for heterochromatin formation involves Suv39h-mediated H3K9me3, which is bound by the heterochromatin protein $\mathrm{HP} 1^{4,10,11}$. HP1 binding promotes compaction and transcriptional repression and stabilizes Suv39h binding to heterochromatin. However, while disruption of Suv39h enzymes results in loss of $\mathrm{H} 3 \mathrm{~K} 9 \mathrm{me} 3$ and dispersion of HP1, the overall organization of heterochromatin in DAPI-dense foci remains largely intact ${ }^{5}$, indicating that other mechanisms are involved.

Several observations suggest a partial redundancy between H3K9 methylation systems. A sub-stoichiometric complex containing G9a, Suv39h, and Eset1 has been described ${ }^{12}$. Eset1 knockdown in Suv39h double-null MEFs results in partial dispersion of DAPI-dense heterochromatic foci ${ }^{13}$. In C. elegans, double deletion of MET-2 (a Setdb1 homolog) and SET-25 (a KMT with some homology to G9a and Suv39h) results in loss of heterochromatin anchoring to the nuclear lamina ${ }^{14}$. Interestingly, MET-2/SET-25 mutant worms are viable despite lacking all H3K9 methylation, but derepress transposons and satellite repeats, leading to genome instability and sterility ${ }^{15,16}$.

We addressed potential redundancy between $\mathrm{H} 3 \mathrm{~K} 9$ methylation systems in mouse fibroblast cells by generating compound mutants for all known SET-domain H3K9 lysine methyltransferases (KMT) using the CRISPR/Cas9 technology. We isolated mouse embryonic fibroblast (MEF) cell lines lacking progressively 2, 4, or all $6 \mathrm{H} 3 \mathrm{~K} 9 \mathrm{KMTs}$. Compound mutant MEF cells lacking all six enzymes lost all H3K9 methylation, derepress multiple families of repeat elements, and display DNA damage and cell death. While mutants retaining the function of some of the $\mathrm{H} 3 \mathrm{~K} 9$-methylating enzymes maintain heterochromatin clustering, 6KO MEF cells show the complete collapse of heterochromatin foci and loss of electron-dense heterochromatin. These results illustrate the functional redundancy between the three H3K9 KMT systems and provide compelling evidence for an essential function of $\mathrm{H} 3 \mathrm{~K} 9$ methylation in heterochromatin organization.

\section{Results}

Gene pair disruptions identify distinct functions for the Suv39h, Eset, and G9a enzymes in directing H3K9 methylation states. In mouse cells, 6 genes encode SET-domain-containing enzymes with a documented methyltransferase activity toward H3K9: Suv39h1 and Suv39h2, Eset1/Setdb1 and Eset2/Setdb2, and G9a/Ehmt2 and Glp/Ehmt1 (Fig. 1a). Since Setdb1/Eset1-deficient cells display growth defects, we took advantage of existing mouse embryonic fibroblasts (MEFs) with a tamoxifen-inducible conditional deletion of Eset $1^{6}$, hereafter called Eset1cKO, or E1c. We refer to the E1c cells as wild-type (WT) control MEF cells, which, after tamoxifen-induced deletion of Eset1, are converted into E1 (Eset1-null) MEFs. We used the CRISPR/Cas9 system in the E1c MEFs to introduce frameshift mutations within the catalytic domain of all the other SET-domain genes encoding H3K9 KMTs (Fig. 1a).

We first generated cell lines lacking the function of each set of paralogous genes. After transfecting E1c MEFs with plasmids carrying Cas9 and the respective guide RNAs, we isolated clones and confirmed the absence of detectable target proteins as well as the efficiency of tamoxifen-induced Eset 1 deletion by western blotting (Fig. 1b). We then assessed the contribution of each enzyme pair to the total levels of H3K9 methylation, by probing chromatin extracts with antibodies specific for H3K9me1, H3K9me2, and H3K9me3 (Fig. 1c). Eset1/Eset2 (E1E2) doublemutant MEFs do not display a significant reduction in overall H3K9 methylation levels. By contrast, G9a/Glp (G9Gl)-deficient cells have greatly lowered $\mathrm{H} 3 \mathrm{~K} 9 \mathrm{me} 1$ and $\mathrm{H} 3 \mathrm{~K} 9 \mathrm{me} 2$, while Suv39h1/Suv39h2 (S1S2) mutants lack most H3K9me3, and to a lesser extent, $\mathrm{H} 3 \mathrm{~K} 9 \mathrm{me} 2$. Thus, the different $\mathrm{H} 3 \mathrm{~K} 9$ enzyme pairs have distinct functions in establishing $\mathrm{H} 3 \mathrm{~K} 9$ methylation, in agreement with published observations ${ }^{5,8}$.

Eset-mutant MEF cells display micronuclei but maintain heterochromatic clustering. We visualized pericentric heterochromatin organization in these mutant MEF cells by performing DNA fluorescence in situ hybridization (DNA FISH) with probes specific for major satellite repeats (MSR). In WT (E1c) control cells, MSR sequences are clustered in bright and regular foci overlapping DAPI-dense areas (Fig. 1d). This organization is maintained in Eset1/Eset2 or G9a/Glp double-deficient cells, and only Suv39h1/Suv39h2 mutant cells display very modest aberrations in the definition of heterochromatic foci.

We then determined the integrity of the nuclear envelope by immunostaining with an antibody directed against the nuclear lamina component Lamin B. We did not observe any alteration in the morphology of the nuclear lamina of G9a/Glp and Suv39h1/ Suv39h2 mutant cells when compared to WT. In contrast, Eset1/ Eset2-deficient cells have multiple micronuclei and display deformation of the nuclear lamina/envelope (Fig. 1e), suggesting that they harbor significant DNA damage.

These data indicate distinct functions for the Suv39h1/ Suv39h2, Eset1/Eset2, and G9a/Glp systems in establishing H3K9 methylation states, as well as the different requirements for maintaining nuclear envelope morphology. However, none of these gene pair mutants show significant alterations in heterochromatin organization, suggesting a higher degree of redundancy between these three H3K9-methylating systems.

Compound KMT-mutant MEF cells that lack the six SETdomain H3K9-methylating enzymes have lost all H3K9 methylation states. To address this question, we next generated mutant cell lines with compound inactivation of multiple $\mathrm{H} 3 \mathrm{~K} 9$ SET-domain genes, in combination with the Eset1-conditional mutation. We proceeded stepwise to establish two series of mutant cell lines that progressively lack increasing numbers of H3K9-methylating enzymes (Fig. 2a). In the first series, we generated Eset1-conditional MEF cells lacking Suv39h1 and Suv39h2 (E1c S1S2) and further inactivated the remaining three $\mathrm{H} 3 \mathrm{~K} 9$ SET-domain genes to generate cells carrying mutations in all six H3K9-methylating enzymes (E1cE2 S1S2 G9Gl). In a parallel series, we first targeted Eset2 (E1cE2), then G9a and Glp (E1cE2 G9Gl), and finally Suv39h1 and Suv39h2 (E1cE2 G9Gl S1S2). We confirmed the absence of mutant gene products, as well as the efficiency of tamoxifen-induced Eset1 deletion by western blotting (Fig. 2b). 

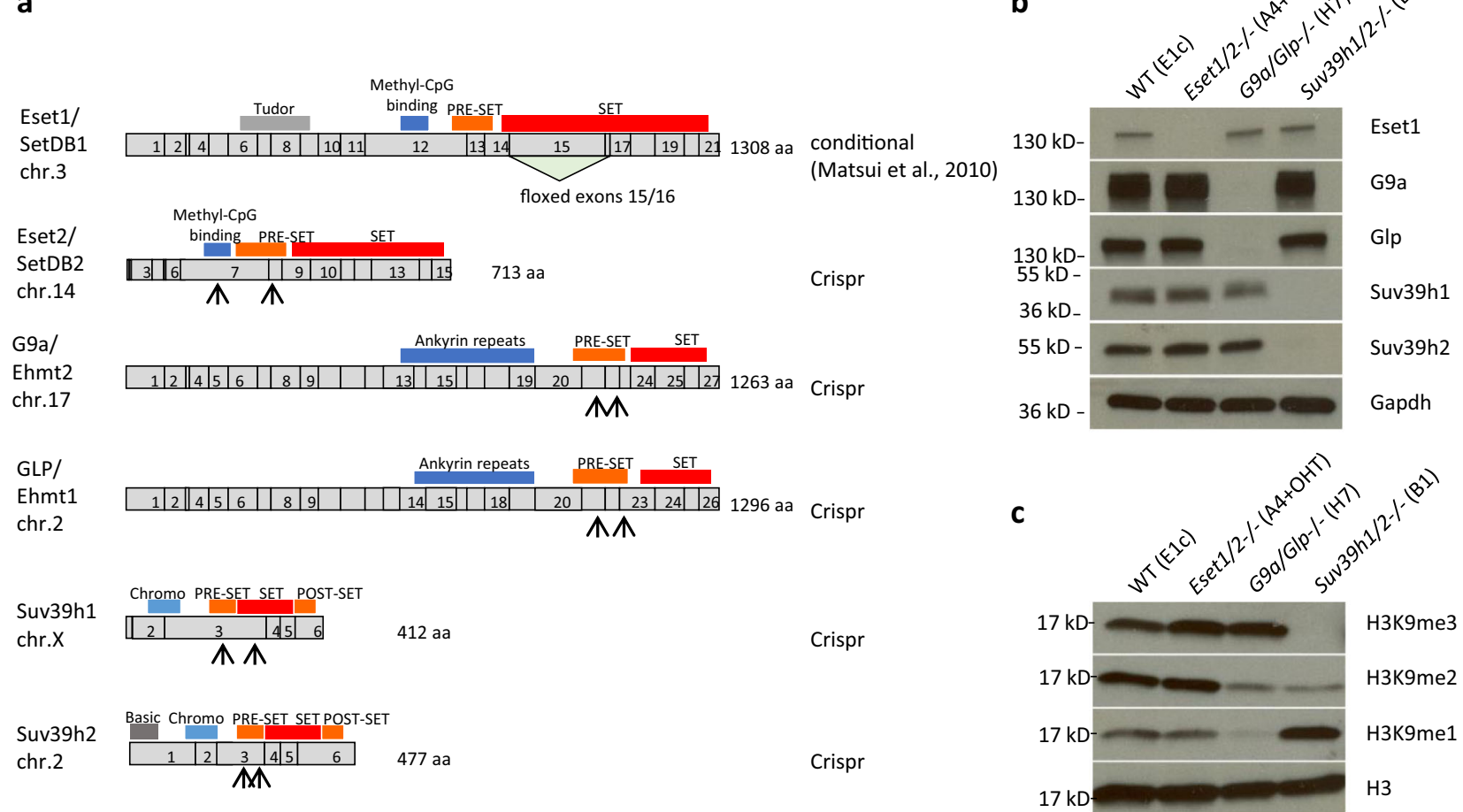

Crispr

Suv39h2 Basic Chromo PRE-SET SET POST-SET

chr.2

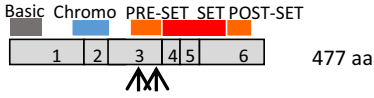

Crispr

$17 \mathrm{kD}$

d

e WT

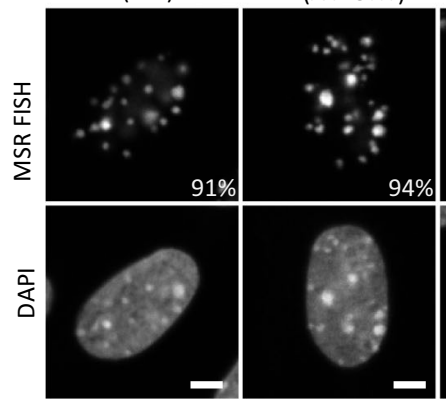

(H7)

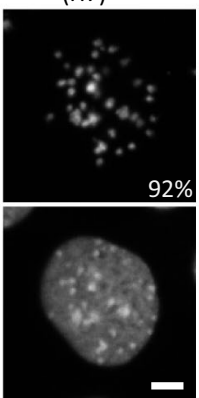

(B1)

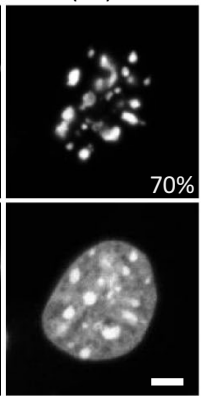

(E1c)

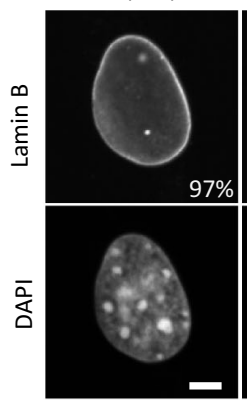

Eset1/2-/(A4+OHT)

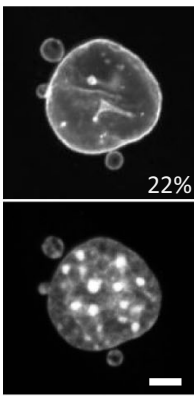

G9a/G/p-/

(H7)

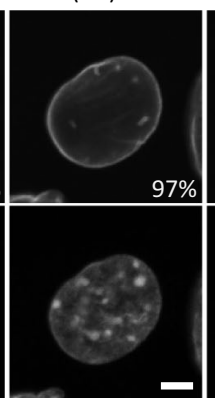

Suv39h1/2-/

(B1)

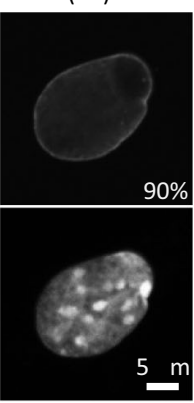

Fig. 1 Three distinct gene pairs encode H3K9 KMT enzymes in the mouse genome. a Domain structure of the six SET-domain H3K9 KMT. For each gene, numbered boxes indicate exons, and conserved protein domains are indicated above the gene structure. The triangle below Eset1 illustrates the floxed exons deleted upon tamoxifen treatment. For the other SET-domain H3K9 KMT genes, disruptions were generated by CRISPR/Cas9 technology, with the location of the gRNAs indicated by arrows below the gene structure. b Gene pair mutants lacking either Eset1/Eset2, G9a/Glp, or Suv39h1/Suv39h2.

Western blots were performed on whole-cell extracts of the mutant clones (top) using specific antibodies indicated on the right. Gapdh was used as a loading control. Eset 1 deletion was probed with extracts after 2 days of induction in culture with 4-hyroxytamoxifen $(4-\mathrm{OHT}, 1 \mu \mathrm{M})$ followed by 2 days in a normal medium. Representative results of two independent experiments. c Western blot analysis of H3K9 methylation states in H3K9 KMT gene pair mutants. Acid-extracted histones were probed with antibodies specific for H3K9me1, H3K9me2, and H3K9me3. Total H3 was used as a loading control. Representative results of three independent experiments. Source data are provided as a Source Data file. d DNA FISH for major satellite repeats (MSR) in control and H3K9 KMT gene pair mutants. Percentages indicate cells that display the shown pattern. $N=325$ cells examined for WT, 329 for Eset1/2-/-, 301 for G9a/Glp-/-, and 384 for Suv39h1/2-/- over two independent experiments. For each image, lower panels show DAPI counterstaining of the same nuclei. e Immunofluorescence for Lamin B, marking the nuclear lamina. Percentages indicate cells that display the shown pattern. $N=402$ cells examined for WT, 439 for Eset1/2-/-, 321 for G9a/Glp-/-, and 410 for Suv39h1/2-/- over two independent experiments. For each image, lower panels show DAPI counterstaining of the same nuclei. Scale bars represent $5 \mu \mathrm{m}$.

We first characterized the growth properties of these compound H3K9 KMT-mutant cell lines. While all compound H3K9 KMT cell lines are viable and proliferating when growing in the absence of tamoxifen (i.e., when Eset1 is still functional), inducing Eset1 deletion in the continuous presence of tamoxifen leads to cell death after a few days (i.e., more than 4 days) in culture (Fig. 2c). This lethality is more pronounced in cells lacking all six H3K9 KMT (E1E2 S1S2 G9Gl or abbreviated as
$6 \mathrm{KO} 1$ and E1E2 G9Gl S1S2 or abbreviated as 6KO2) when compared to the single inactivation of Eset1 (E1).

For the following experiments and sample preparations, we, therefore, induced Eset 1 deletion by a 2-day incubation with tamoxifen, followed by a 2-day culture in the normal medium (Fig. 2d).

We then examined H3K9 methylation in chromatin extracts from the various H3K9 KMT mutants by western blotting 
a

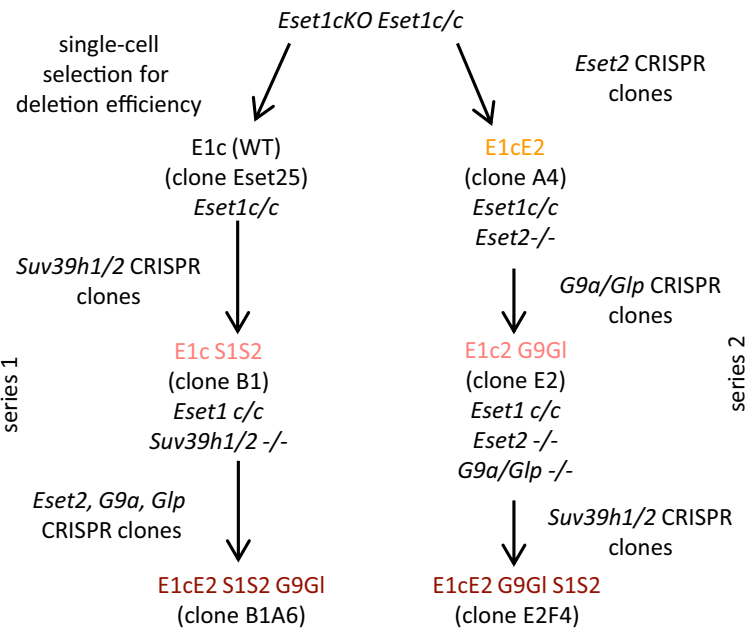

b

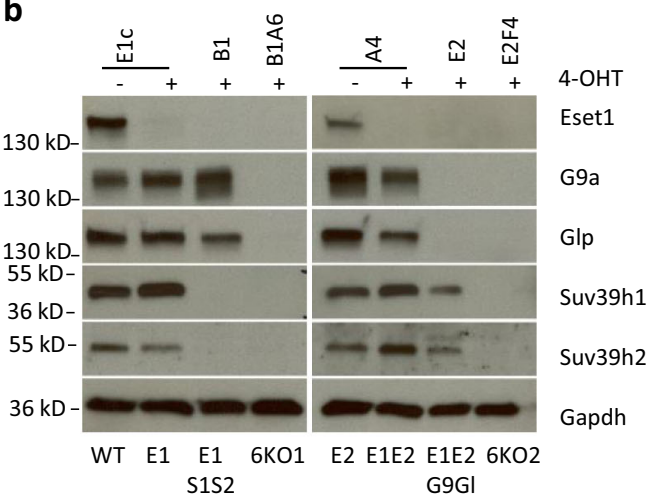

(Eset1 c/c, Eset2 crispr; Suv39h1 crispr, Suv39h2 crispr; G9a crispr, Glp crispr)

C

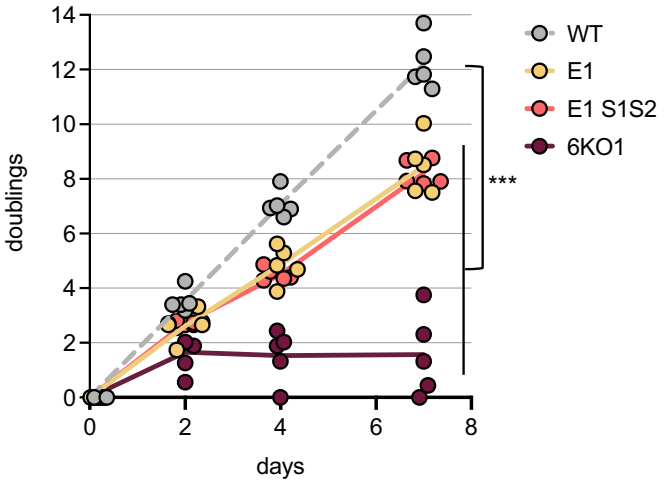

d

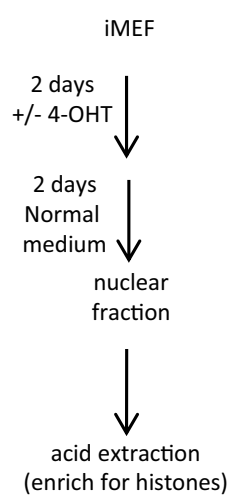

e

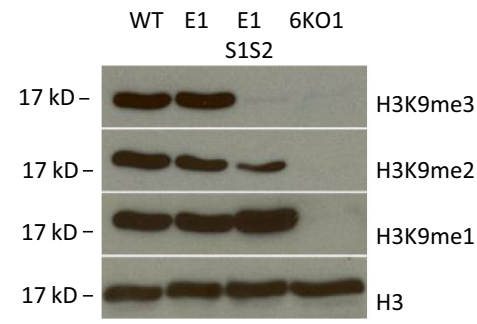

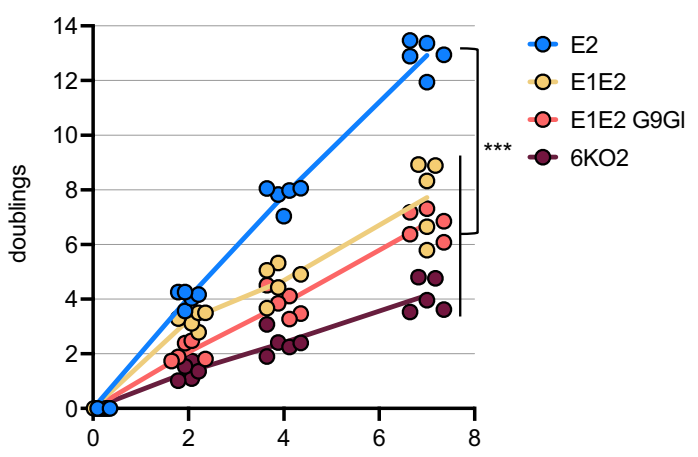

E2 E1E2 E1E2 6KO2 G9GI

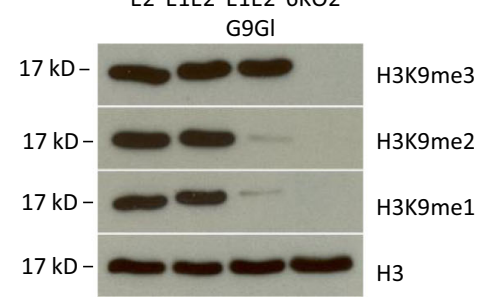

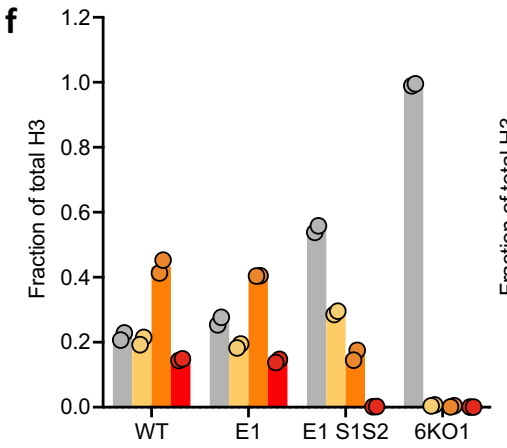

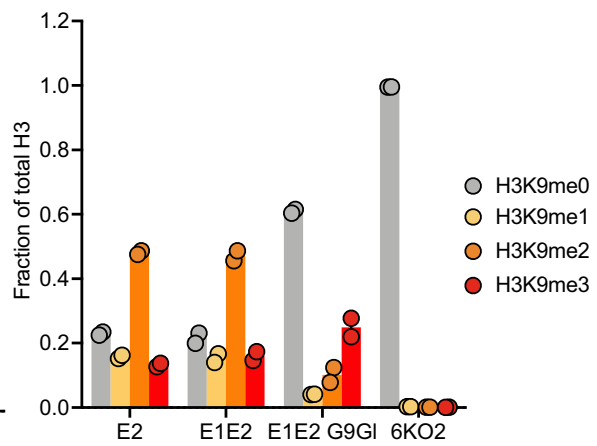

(Fig. 2e). Consistent with our previous observations with H3K9 SET-domain gene pair mutants, Eset1 (E1), Eset2 (E2), and Eset1/ Eset2 (E1E2)-deficient cells display comparable levels of $\mathrm{H} 3 \mathrm{~K} 9$ methylation as WT cells. Compound Eset1 and Suv39h1/Suv39h2 mutants (E1 S1S2) largely lack H3K9me3, while compound Eset1/Eset2 and G9a/Glp mutants (E1E2 G9Gl) have drastically reduced $\mathrm{H} 3 \mathrm{~K} 9 \mathrm{me} 1$ and $\mathrm{H} 3 \mathrm{~K} 9 \mathrm{me} 2$. Strikingly, in extracts from the compound $6 \mathrm{KO} 1$ and $6 \mathrm{KO} 2$ mutants, we could not detect any residual $\mathrm{H} 3 \mathrm{~K} 9 \mathrm{me} 1, \mathrm{H} 3 \mathrm{~K} 9 \mathrm{me}$, or $\mathrm{H} 3 \mathrm{~K} 9 \mathrm{me} 3$.

To validate this observation with an antibody-independent approach, we analyzed the same extracts by mass spectrometry (Fig. 2f). We detected very similar changes in $\mathrm{H} 3 \mathrm{~K} 9$ methylation states, and confirmed the nearly total absence of $\mathrm{H} 3 \mathrm{~K} 9$ methylation in chromatin extracted from the $6 \mathrm{KO} 1$ and $6 \mathrm{KO} 2$ 
Fig. 2 Loss of H3K9 methylation in compound H3K9 KMT-mutant MEF cells. a Generation of compound H3K9 KMT-mutant MEF by sUccessive CRISPR/ Cas9-mediated inactivation of genes encoding H3K9 KMT enzymes. Two series of H3K9 KMT mutants were generated. Nomenclature for the H3K9 KMT mutants is indicated on the scheme. E1c (Eset7-conditional, also WT), E1 (Eset1-null), E2 (Eset2-null), G9 (G9a-null), GI (Glp-null,) S1 (Suv39h1-null), and S2 (Suv39h2-null). b Western blot analysis for H3K9 KMT gene products in whole-cell extracts of WT and H3K9 KMT mutants. Gapdh was used as a loading control. Representative results of two independent experiments. Source data are provided as a Source Data file. c Proliferation capacity of compound H3K9 KMT mutants. The $y$ axis indicates cumulative doublings after consecutive days in culture ( $x$ axis). E1c/WT and E2 were grown in a normal medium, and all other H3K9 KMT mutants in presence of $1 \mu \mathrm{M} 4-\mathrm{OHT}$ in a culture medium (individual data points with line connecting their mean, $N=5$ independent experiments). Statistically significant differences in doubling time, when compared to WT (left) or E2 (right) growth curves are shown by asterisks ( ${ }^{\star \star \star} P=$ $1.1 \times 10^{-10}$ for $E 1$ and $E 1$ S1S2, $P=7.2 \times 10^{-11}$ for $6 K O 1, P=2.8 \times 10^{-8}$ for E1E2, $P=2.6 \times 10^{-8}$ for E1E2 G9GI and $6 \mathrm{KO}$, extra-sum-of-squares $F$ test). d Induction of Eset1 deletion. Cells were grown for 2 days in presence of $1 \mu \mathrm{M} 4-\mathrm{OHT}$ in culture medium, followed by 2 days in normal medium without 4 $\mathrm{OHT}$. After cell lysis and fractionation, histones were acid-extracted from the nuclear fraction. e Western blot analysis of H3K9 methylation states in compound H3K9 KMT mutants. Acid-extracted histones were probed with antibodies specific for H3K9me1, H3K9me2, and H3K9me3. Total H3 was used as a loading control. Representative results of three independent experiments. Source data are provided as a Source Data file. $\mathbf{f}$ Mass spectrometry analysis of $\mathrm{H} 3 \mathrm{~K} 9$ methylation states. The $y$ axis indicates the fraction of total $\mathrm{H} 3$ carrying $\mathrm{H} 3 \mathrm{~K} 9 \mathrm{me1}, \mathrm{H} 3 \mathrm{~K} 9 \mathrm{me} 2$, or H3K9me3 modifications, determined by normalizing intensities obtained for the corresponding peptides in nanoLC-MS (bars represent mean with individual data points overlaid, $N=$ two independent samples).

mutants, with H3K9me1, H3K9me2, and H3K9me3 levels reduced to $\leq 1 \%$ as compared to $\mathrm{WT}$.

We also investigated possible changes in the abundance of other histone modifications by mass spectrometry, and observed only a moderate increase in $\mathrm{H} 3 \mathrm{~K} 9$ acetylation or in $\mathrm{H} 3 \mathrm{~K} 27 \mathrm{me} 3$, together with a decrease in $\mathrm{H} 3 \mathrm{~S} 10$ phosphorylation, specifically in extracts from the $6 \mathrm{KO}$ mutants (Supplementary Fig. 1A). We also examined changes in total levels of $\mathrm{H} 3$ or $\mathrm{H} 4$ acetylation, as well as $\mathrm{H} 3 \mathrm{~K} 4 \mathrm{me} 3$ by western blotting. While $\mathrm{H} 3$ acetylation was increased in some of the H3K9 KMT mutants, particularly in extracts from $6 \mathrm{KO}$ cells, $\mathrm{H} 4$ acetylation and $\mathrm{H} 3 \mathrm{~K} 4 \mathrm{me} 3$ were not significantly altered (Supplementary Fig. 1B). Therefore, loss of H3K9 methylation does not lead to a general or bulk increase in histone modifications associated with active chromatin, although the absence of $\mathrm{H} 3 \mathrm{~K} 9$ methylation might facilitate $\mathrm{H} 3$ acetylation. Importantly, these changes may not occur specifically at heterochromatin regions.

The presence of micronuclei in some of the H3K9 KMT mutants prompted us to examine the phosphorylation of the histone variant H2A.X ( $\gamma$ H2A.X), a known marker of DNA double-strand breaks ${ }^{17}$. We observed an increase in $\gamma \mathrm{H} 2 \mathrm{~A} . \mathrm{X}$ signal in some of the H3K9 KMT mutants, particularly in extracts from 6KO1 cells (Supplementary Fig. 1C), but these levels remained low when compared to control cells treated with the topoisomerase inhibitor Etoposide and they were not apparent in extracts from $6 \mathrm{KO} 2$ cells. This might reflect different kinetics of DNA repair at heterochromatin regions and/or a somewhat altered involvement of heterochromatin proteins in the activation of DNA repair pathways ${ }^{18}$ between the $6 \mathrm{KO} 1$ and $6 \mathrm{KO} 2$ mutants. In addition, it has been shown that micronuclei can also form without $\gamma$ H2A.X accumulation ${ }^{19}$.

We further assessed global DNA methylation levels in the $6 \mathrm{KO}$ mutant cells, either by DNA dot-blot analysis using an antibody recognizing 5-methylcytosine $(5 \mathrm{mC})$ (Supplementary Fig. 1D) or by mass spectrometry (Supplementary Fig. 1E). As controls, we used mouse ES cells that were either wild type or mutant for the three DNA methyltransferases establishing $5 \mathrm{mC}(\text { Dnmt TKO })^{20}$. Dnmt TKO ES cells have only residual levels of $5 \mathrm{mC}$ and of 5hydroxymethylcytosine $(5 \mathrm{hmC})$. In contrast, $6 \mathrm{KO} \mathrm{MEF}$ cells maintain high, although modestly decreased $5 \mathrm{mC}$ and $5 \mathrm{hmC}$ levels, corresponding to $\sim 80 \% 5 \mathrm{mC}(P<0.05)$ or $60 \% 5 \mathrm{hmC}$ (non-significant) of the signals observed in WT MEFs. These data indicate that loss of $\mathrm{H} 3 \mathrm{~K} 9$ methylation does not lead to an immediate and overall or genome-wide loss of DNA methylation.

Massive derepression of repeat elements and retrotransposons in compound H3K9 KMT-mutant MEF cells. We determined the transcriptional response of $\mathrm{H} 3 \mathrm{~K} 9 \mathrm{KMT}$ disruption by performing RNA sequencing (RNA-seq) in the various H3K9 KMTmutant cell lines. We first focused on all repetitive elements in the mouse genome. As previously reported ${ }^{6,21}$, deletion of Eset 1 alone is sufficient to induce derepression of a subset of endogenous retroviruses (ERV) (Fig. 3b and Supplementary Fig. 2). Notably, the numbers of derepressed ERV elements, as well as the magnitude of their upregulation, are increased in cell lines where additionally also $G 9 a$ and $G l p$ are deleted (E1E2 G9Gl) and further augmented in the $6 \mathrm{KO} 1$ and $6 \mathrm{KO} 2$ cells (Fig. 3a, b). Among the distinct ERV subtypes, ERV class I elements of the ERV1 family are most sensitive to Eset1 depletion (Supplementary Fig. 2A). ERV class II elements, such as the ERVK family, show only a modest increase in expression in cells lacking Eset1, or both Eset1 and Eset2 (E1E2), but are strongly upregulated in E1E2 $\mathrm{G} 9 a \mathrm{Gl}$ mutants and in $6 \mathrm{KO} 1$ and $6 \mathrm{KO} 2$ cells (Fig. 3c). In contrast, expression of ERV class III elements is either unaltered (ERVL), or only moderately increased (MaLR), even in mutant cells lacking all six KMT (Supplementary Fig. 2A).

Non-long terminal repeat (LTR) containing retrotransposons are classified as short or long interspersed nuclear elements (SINE or LINE). While expressed at relatively high levels in WT cells, SINE is not dysregulated by the lack of H3K9 KMT (Fig. 3b). LINE elements, in particular LINE L1, are overexpressed specifically in H3K9 KMT-mutant cells lacking $G 9 a$ and Glp (E1E2 G9Gl) and in 6KO1 and 6KO2 cells (Fig. 3a, b).

Satellite DNA forms large tandem repeat arrays at centromeric and pericentromeric regions, but can also be found in a few intergenic regions. In particular, major satellite repeats (MSR) are repressed by Suv39h enzymes in various cell types, including fibroblasts ${ }^{22}$. Satellite repeats are exclusively derepressed in mutant cells lacking Suv39h enzymes, and not in other compound H3K9 KMT mutants (Fig. 3b). In cells depleted for Suv39h1/Suv39h2 together with Eset1, the only upregulated satellite repeats are MSR (annotated as GSAT_MM in Supplementary Fig. 2A). Interestingly, MSR expression is further increased in cells that lack all $6 \mathrm{KMT}$, indicating that while Suv39h are the chief H3K9-methylating enzymes for MSR repression, other $\mathrm{H} 3 \mathrm{~K} 9 \mathrm{KMT}$ can participate in their control (Fig. 3c and Supplementary Fig. 2A). In addition to MSR, 6KO1 and $6 \mathrm{KO} 2$ mutant cells also upregulate MMSAT4 sequences (Supplementary Fig. 2A).

We also assessed changes in chromatin accessibility by ATAC-seq. The disruption of H3K9 KMT leads to a global increase in chromatin accessibility over repeat elements, which is particularly pronounced for MSR sequences (Fig. 3c, d and Supplementary Fig. 2B). Surprisingly, however, changes in 
a

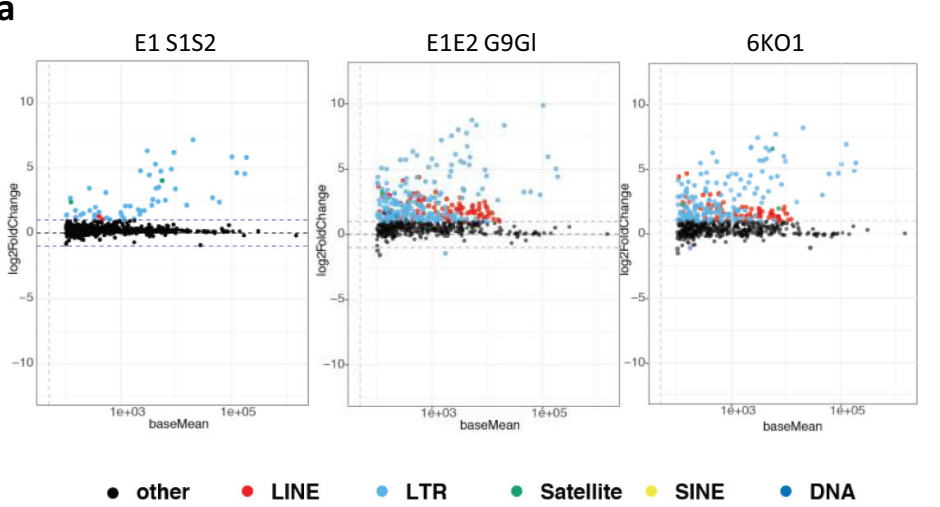

b

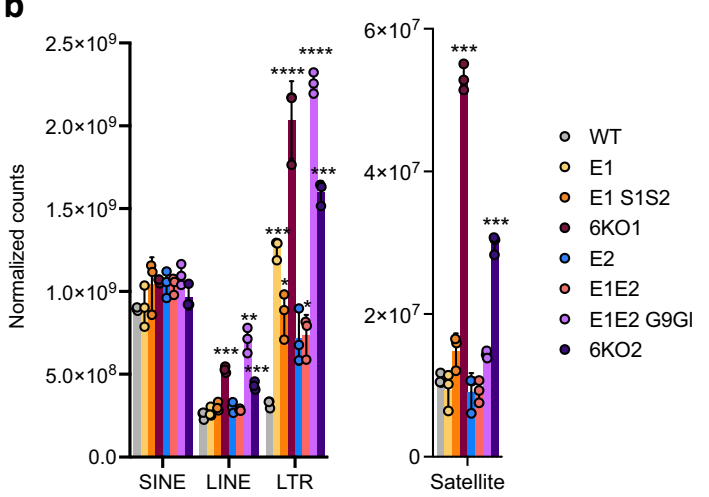

C

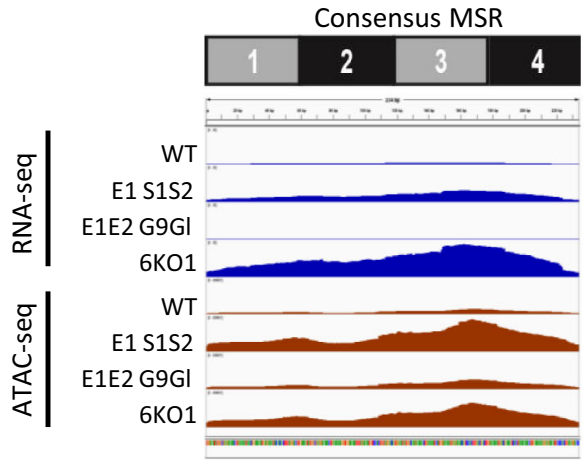

d

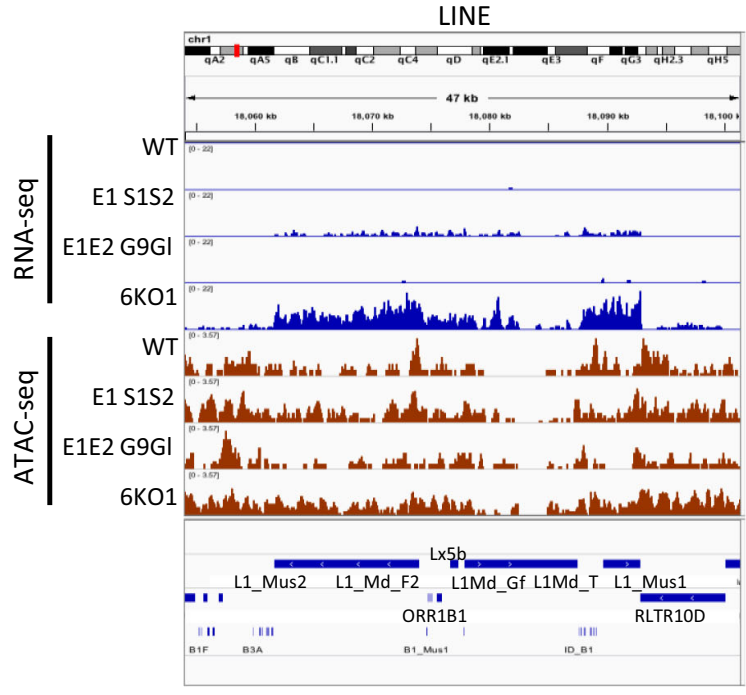

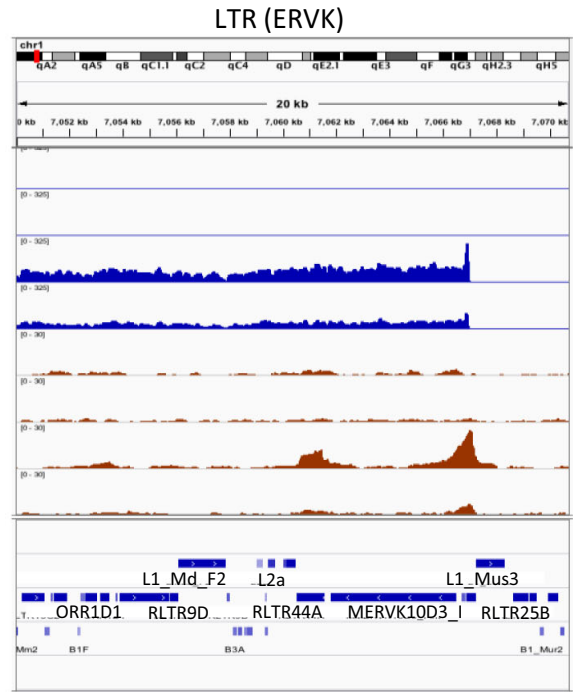

Fig. 3 Massive derepression of repeat elements in compound HзK9 KMT-mutant MEF cells. a MA plots depicting changes in repeat element expression in compound H3K9 KMT mutants relative to WT, as determined by RNA sequencing (RNA-seq). From left to right: E1 S1S2, E1E2 G9GI, and 6KO1. Black dots represent repeat elements whose change in expression is not statistically significant. Red, LINE elements. Light blue, LTR retrotransposons. Green, Satellites. Yellow, SINE elements. Dark blue, DNA transposons. b Global changes in expression for distinct repeat classes (SINE, LINE, LTR, and Satellite), in each of the compound H3K9 KMT mutants. The $y$ axis indicates normalized read counts (mean \pm SD, individual data points overlaid, $N=3$ independent samples). Satellites are shown on a separate graph due to their lower expression level. Asterisks indicate statistically significant differences when compared to WT levels (LINE: ${ }^{\star \star \star} P=1.1 \times 10^{-4}$ for $6 \mathrm{KO} 1{ }^{* \star} P=0.006$ for E1E2 G9GI, ${ }^{\star \star \star} P=8.0 \times 10^{-4}$ for $6 \mathrm{KO}$; LTR: ${ }^{\star \star \star} P=3.7 \times 10^{-4}$ for $E 1$, ${ }^{\star} P=0.019$ for $E 1$ S1S2, ${ }^{\star \star \star \star} P=6 \times 10^{-5}$ for $6 K O 1,{ }^{\star} P=0.026$ for E1E2, ${ }^{\star \star \star \star} P=8.4 \times 10^{-5}$ for E1E2 G9G1, ${ }^{\star \star \star} P=3.6 \times 10^{-4}$ for $6 \mathrm{KO}$; Satellite, ${ }^{\star \star \star} P=1.2 \times 10^{-4}$ for $6 \mathrm{KO} 1$ and $P=1.4 \times 10^{-4}$ for $6 \mathrm{KO}$, two-sided, unpaired $t$ test). c Coverage plot of major satellite repeat (MSR) transcripts to the consensus MSR sequence in WT and H3K9 KMT mutants (from top to bottom: E1 S1S2, E1E2 G9GI, and 6KO1). ATAC-seq indicating DNA accessibility in the same mutants is shown below. d Genome browser tracks showing expression levels (top) and ATAC-seq profiles (bottom) of example genomic regions with repeat elements. Left, genomic region harboring LINE elements. Right, region with LTR-containing elements (ERVK). 
a

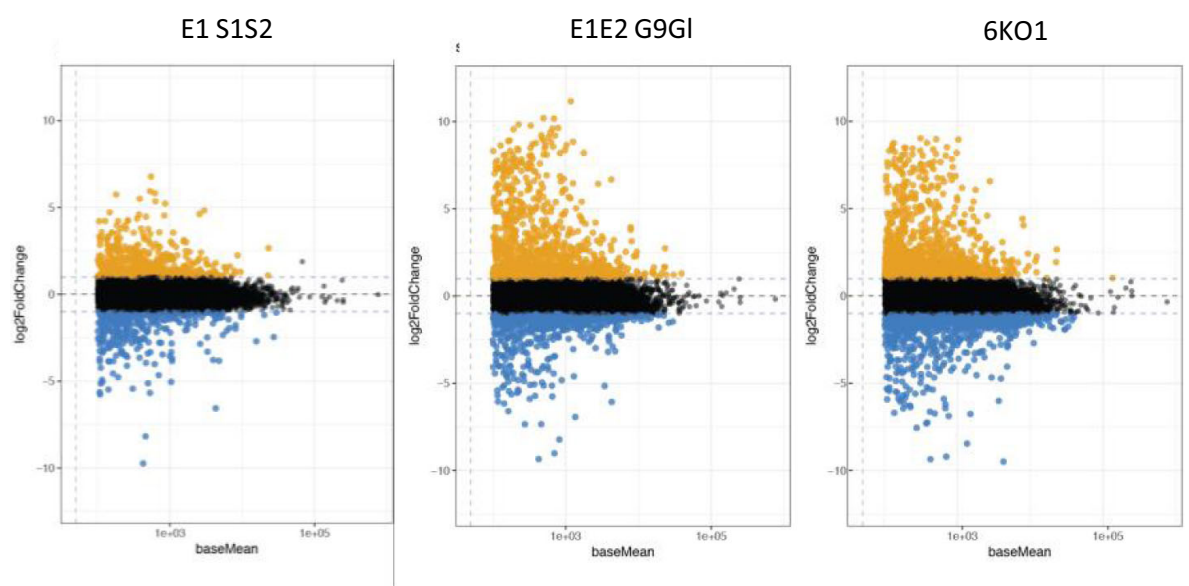

b

E1 S1S2

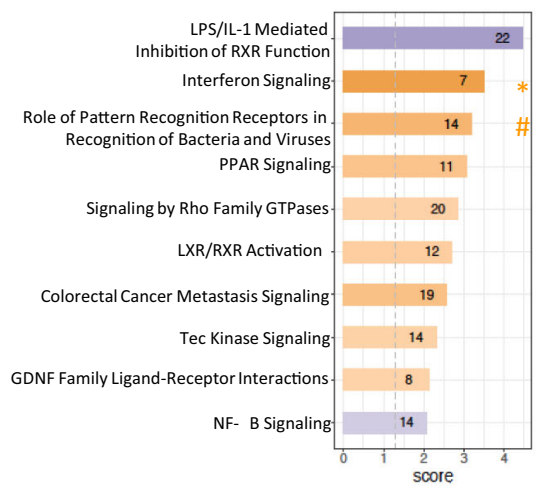

* Interferon signaling
E1E2 G9GI

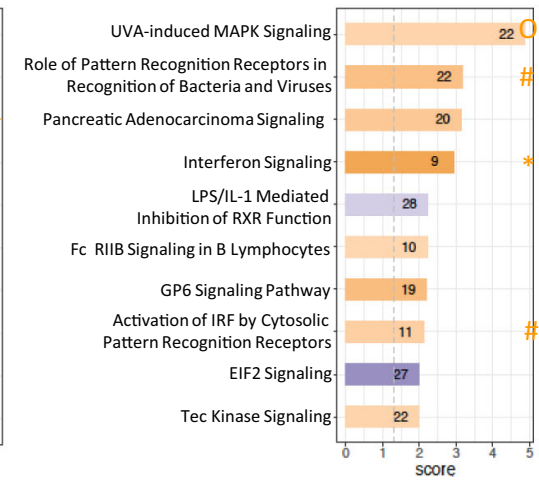

O MAPK signaling
$6 \mathrm{KO} 1$

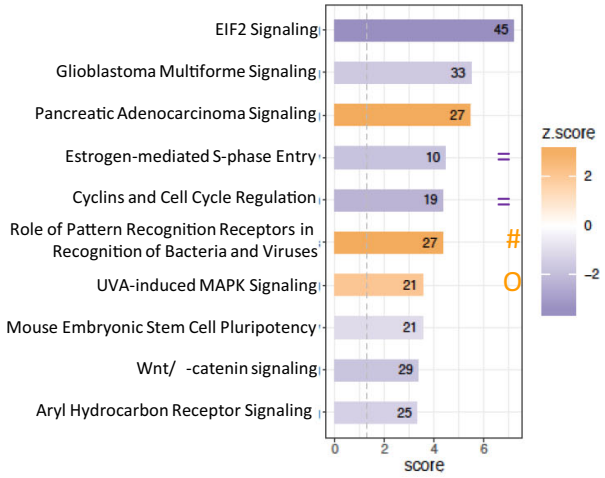

$=$ Cell cycle

C

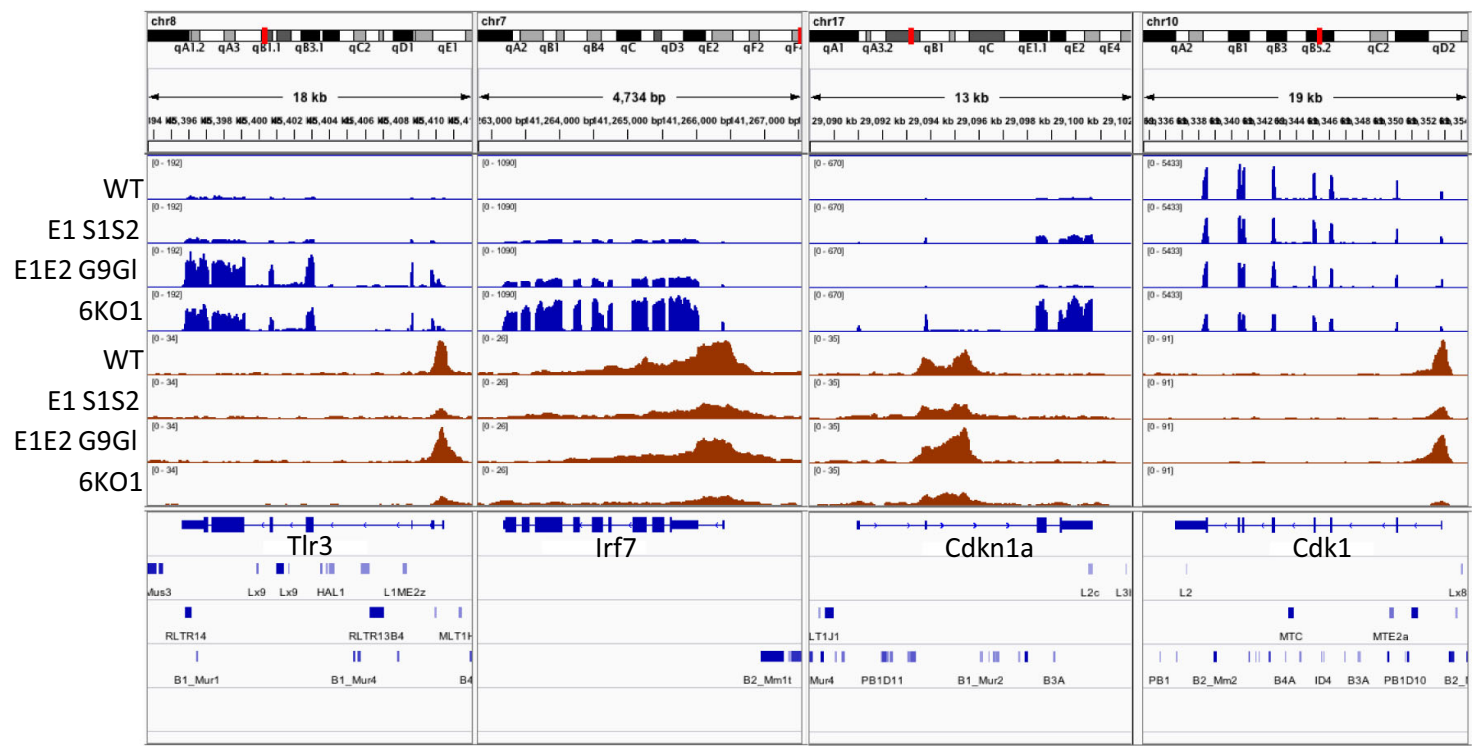

chromatin accessibility did not strictly reflect changes in repeat expression, and several repeat types, such as ERVL, gained chromatin accessibility without being derepressed (Supplementary Fig. 2B).

In summary, the disruption of H3K9 KMT leads to the derepression of multiple families of repeat elements. These results also reveal that distinct $\mathrm{H} 3 \mathrm{~K} 9 \mathrm{KMT}$ have both specific and overlapping functions in the silencing of repeat elements.

Activation of pattern-recognition and DNA damage pathways in compound H3K9 KMT-mutant MEF cells. We next analyzed 
Fig. 4 Changes in gene expression in compound H3K9 KMT-mutant MEF cells. a MA plots depicting changes in gene expression in compound H3K9 KMT mutants relative to WT, as determined by RNA sequencing (RNA-seq). From left to right: E1 S1S2, E1E2 G9Gl, and 6KO1. Black dots represent genes whose changes in expression are not statistically significant. Yellow dots represent upregulated genes, and blue dots downregulated genes. $\mathbf{b}$ Ingenuity pathway analysis (IPA) of genes differentially expressed in compound H3K9 KMT mutants relative to WT. From left to right: E1 S1S2, E1E2 G9GI, and 6KO1. The $x$ axis indicates fold change, and the color code indicate the $z$ score for each category (legend on the right). Orange indicates upregulation, and purple downregulation of the pathway. Pathways related to pattern-recognition receptors, interferon signaling, MAP kinase signaling, or cell cycle regulation are highlighted by symbols as indicated below the table. c Genome browser tracks showing RNA expression levels (top) or ATAC accessibility (bottom) of example genes in compound H3K9 KMT mutants. From left to right, Toll-like receptor 3 (T/r3), interferon regulatory factor 7 (Irf7), cyclin-dependent kinase inhibitor $1 \mathrm{~A}(\mathrm{Cdkn1a)}$, and cyclin-dependent kinase 1 (Cdk1). For both RNA-seq and ATAC-seq tracks, the top profile corresponds to WT, followed from top to bottom by E1 S1S2, E1E2 G9GI, and 6KO1 mutants.

changes in gene expression levels upon loss of H3K9 methylation. We observed dysregulation of increasing numbers of genes in H3K9 KMT mutants lacking progressively more H3K9 KMT (Fig. 4a). While Eset1 mutant cells display 362 upregulated and 331 downregulated genes (fold change $\geq 2, P$ value $\leq 0.01$ ), when compared to WT control cells, cells depleted for all six KMT show deregulation of thousands of genes (3427 up and 1632 down in $6 \mathrm{KO} 1$ and 2517 up and 897 down in 6KO2).

We determined the functional classification of these deregulated genes with the Ingenuity Pathway Analysis database (Fig. 4b). Among the most significantly enriched pathways, pattern-recognition receptors were consistently upregulated. This included, among other genes, the Tlr3 and Tlr4 genes, encoding Toll-like receptors, as well as Ddx58/Rig-I (Fig. 4c). This is consistent with previous studies where treatment of cancer cells with DNMT inhibitors led to overexpression of ERV, which triggered the activation of the double-stranded RNA sensor TLR3 and, in turn, a type I interferon response ${ }^{23,24}$. Accordingly, H3K9 KMT-deficient MEF cells also display activation of interferon signaling (significantly enriched pathway in all H3K9 KMTmutant cells except Eset2 mutants), including overexpression of type I interferon regulators Irf7, Irf9, and Stat1 (Fig. 4c).

Other significantly altered pathways indicate activation of DNA damage-sensing mechanisms. This includes MAP kinase signaling, with upregulation of Mapk10, Mapk11, and Pik3r5, among others, and of the p53 pathway, with increased expression of Trp53inp1, Rb1, and Cdkn1a (Fig. 4c). In agreement with the growth defect exhibited by the H3K9 KMT-mutant cell lines, we also observed reduced expression of multiple cyclin-dependent kinases (Cdk1,Cdk2, Cdk4, Cdk6, and Cdk7, Fig. 4c), and upregulation of apoptosis-related factors, such as Casp7 and multiple poly (ADP-ribose) polymerases.

Collectively, these results indicate that loss of H3K9 KMT induces a signaling cascade, including DNA damage-sensing pathways, interferon response, attenuation of cell proliferation, and cell death. The activation of pattern-recognition receptors, such as Tlr3 and Rig-I, suggests that a major cause of these defects could be the massive derepression of repeat elements, primarily ERV, that we observed in the H3K9 KMT-mutant cells.

Dysregulation of H3K9me-positive vs. H3K9me-negative genes in H3K9 KMT-mutant MEF cells. We wondered to what extent these changes in gene expression would reflect direct participation of H3K9 methylation. Since comprehensive datasets for all three H3K9 methylation states are not available in mouse fibroblasts, we generated reference maps for H3K9me1, H3K9me2, and $\mathrm{H} 3 \mathrm{~K} 9 \mathrm{me} 3$ in WT (E1c) MEFs. We first validated the antibodies by ChIP-qPCR at known target loci (Supplementary Fig. 3A) and then performed chromatin immunoprecipitation followed by deep sequencing (ChIP-seq). Peak calling identified 42893 regions of enrichment for H3K9me1, 29285 for H3K9me2, and 27281 for H3K9me3. Distinct H3K9 methylation states were often observed in overlapping genomic intervals. However,
H3K9me1 peaks had only low signals for $\mathrm{H} 3 \mathrm{~K} 9 \mathrm{me} 2$ and H3K9me3, H3K9me2 peaks coincided with broad signals for H3K9me3 and H3K9me3 peaks also had some enrichment for H3K9me2 (Supplementary Fig. 3B, top panels). In total, $559 \mathrm{Mb}$, corresponding to $21 \%$ of the mouse genome were decorated by H3K9 methylation in WT MEFs.

A large fraction of $\mathrm{H} 3 \mathrm{~K} 9$ methylated regions corresponded to intergenic and intronic sequences, ranging from $55 \%$ for $\mathrm{H} 3 \mathrm{~K} 9$ me1 to 69\% for H3K9me2 and 70\% for H3K9me3 (Supplementary Fig. 3C). A smaller subset of regions overlapped with gene promoters, defined as sequences within $+/-1$ kilobase $(\mathrm{kb})$ of the transcription start site (TSS). This was the case for $19 \%$ of $\mathrm{H} 3 \mathrm{~K} 9 \mathrm{mel}$ and around $10 \%$ for H3K9me2 or H3K9me3-enriched regions. We identified 4289 genes that are decorated by $\mathrm{H} 3 \mathrm{~K} 9 \mathrm{mel}$ at their promoters (e.g., Tcap and Cacna1i), 1567 genes with H3K9me2-positive promoters (e.g., Defb40 and Olfr1431), and 1415 genes with promoters enriched for H3K9me3 (e.g., Nkx6-1 and Bmp2) (see Supplementary Fig. 4A). The majority of H3K9me1-positive promoters (3179 genes) were devoid of H3K9me2 and H3K9me3. By contrast, H3K9me2 and H3K9me3 often decorated the same gene promoters and only 270 or 462 genes were predominantly marked by $\mathrm{H} 3 \mathrm{~K} 9 \mathrm{me} 2$ or $\mathrm{H} 3 \mathrm{~K} 9 \mathrm{me} 3$ (Supplementary Fig. 4B). In total, we detected $\mathrm{H} 3 \mathrm{~K} 9$ methylation at the promoters of 5297 genes.

We then used our RNA-seq data to determine the expression status of these $\mathrm{H} 3 \mathrm{~K} 9$ me-positive genes in WT (E1c) MEFs and in the various H3K9 KMT-mutant cell lines. For this, we applied a meta-analysis that illustrates gene expression in violin plots. Surprisingly, genes with $\mathrm{H} 3 \mathrm{~K} 9$ mel-marked promoters have significantly $\left(P<2.2 \times 10^{-16}\right)$ higher expression levels as compared to genes without $\mathrm{H} 3 \mathrm{~K} 9$ methylation $(\mathrm{H} 3 \mathrm{~K} 9 \mathrm{me} 0$ genes in the left panel of Fig. 5a), and this elevated expression was largely unaltered in all of the H3K9 KMT mutants (Fig. 5a and browser shot for example gene $B b s 9$ in Fig. 5b). By contrast and as expected, genes with $\mathrm{H} 3 \mathrm{~K} 9 \mathrm{me} 2-$ or $\mathrm{H} 3 \mathrm{~K} 9 \mathrm{me} 3$-marked promoters were silenced and had significantly $\left(P<2.2 \times 10^{-16}\right)$ reduced or no expression levels as compared to $\mathrm{H} 3 \mathrm{~K} 9 m e-n e g a t i v e$ (H3K9me0) genes (Fig. 5a). The H3K9me2 mediated gene silencing was weakened $(P<0.01)$ in E1E2 G9Gl compound H3K9 KMT-mutant cells (Fig. 5a). Both H3K9me2 and H3K9me3 mediated gene silencing was partly lost $(P<0.001)$ in $6 \mathrm{KO} 1$ and $6 \mathrm{KO} 2$ cells (Fig. $5 \mathrm{a}$ and browser shots for example genes Treml4 and Zfp 936 in Fig. 5b). However, it is important to note that loss of $\mathrm{H} 3 \mathrm{~K} 9$ methylation only caused desilencing in a minor fraction of the $\mathrm{H} 3 \mathrm{~K} 9 \mathrm{me} 2-$ or $\mathrm{H} 3 \mathrm{~K} 9 \mathrm{me} 3$-positive genes: with a twofold cutoff (and a base mean of $>100$ reads), 226 of 270 H3K9me2-positive genes, and 390 of 462 H3K9me3-positive genes were not significantly upregulated in any of the H3K9 KMT mutants, not even in the $6 \mathrm{KO} 1$ or $6 \mathrm{KO} 2$ cells.

We further focused on the $6 \mathrm{KO}$ mutants and analyzed changes in gene expression (as compared to WT) separately for H3K9mepositive and for H3K9me-negative genes (H3K9me+ and H3K9me- genes, Fig. 5c). While H3K9me-positive genes represent $11 \%$ (5297 genes) of all genes, they account for $>20 \%$ 
a

H3K9me0 (42626 genes)

H3K9me1 (3179 genes)

H3K9me2 (270 genes)

H3K9me3 (462 genes)
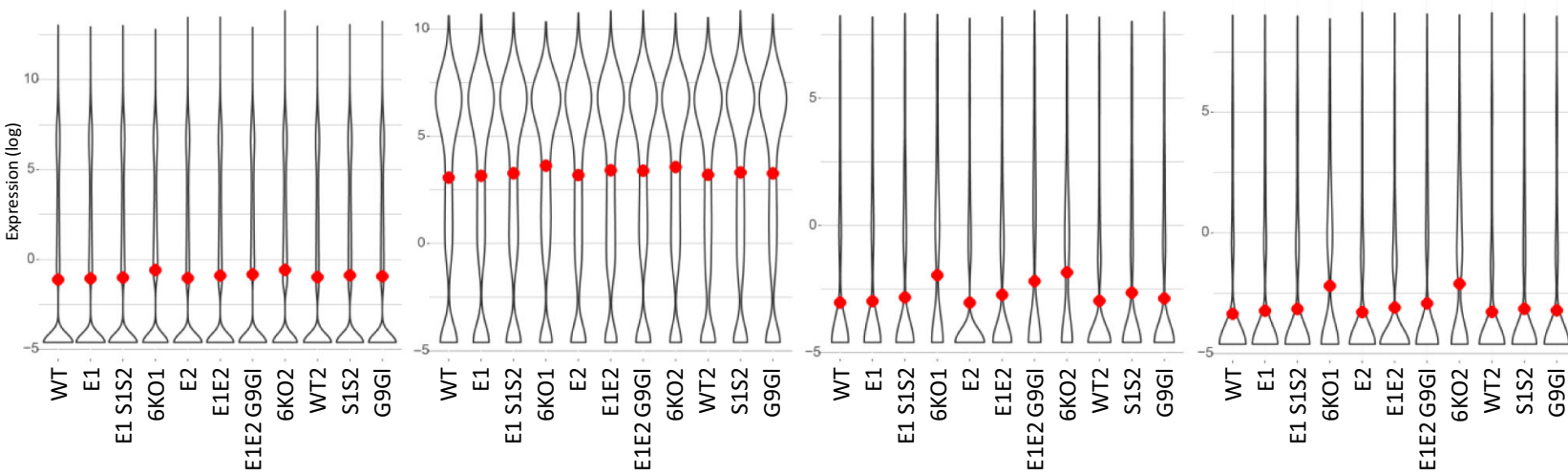

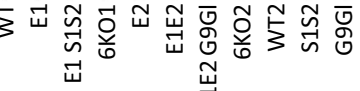
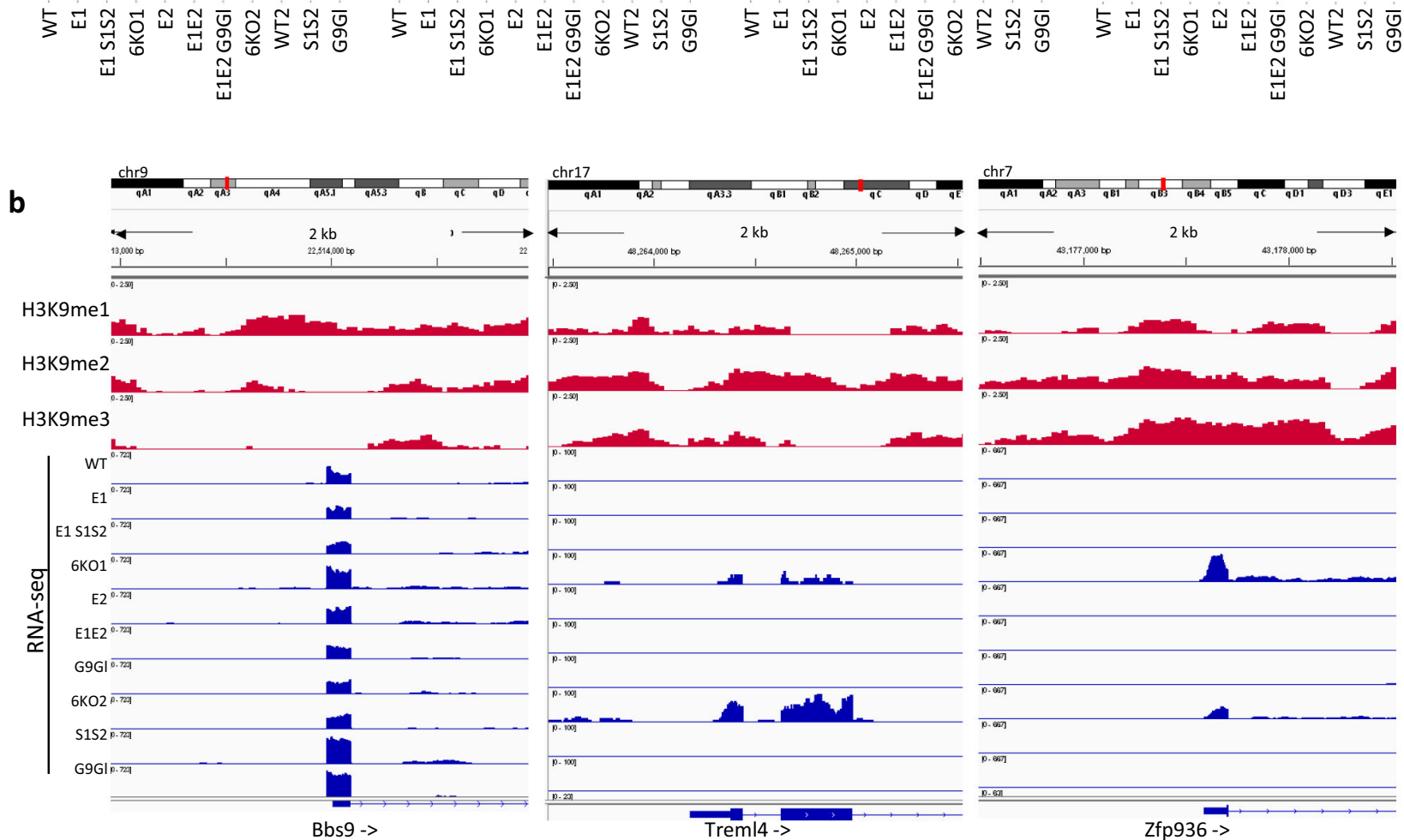

C
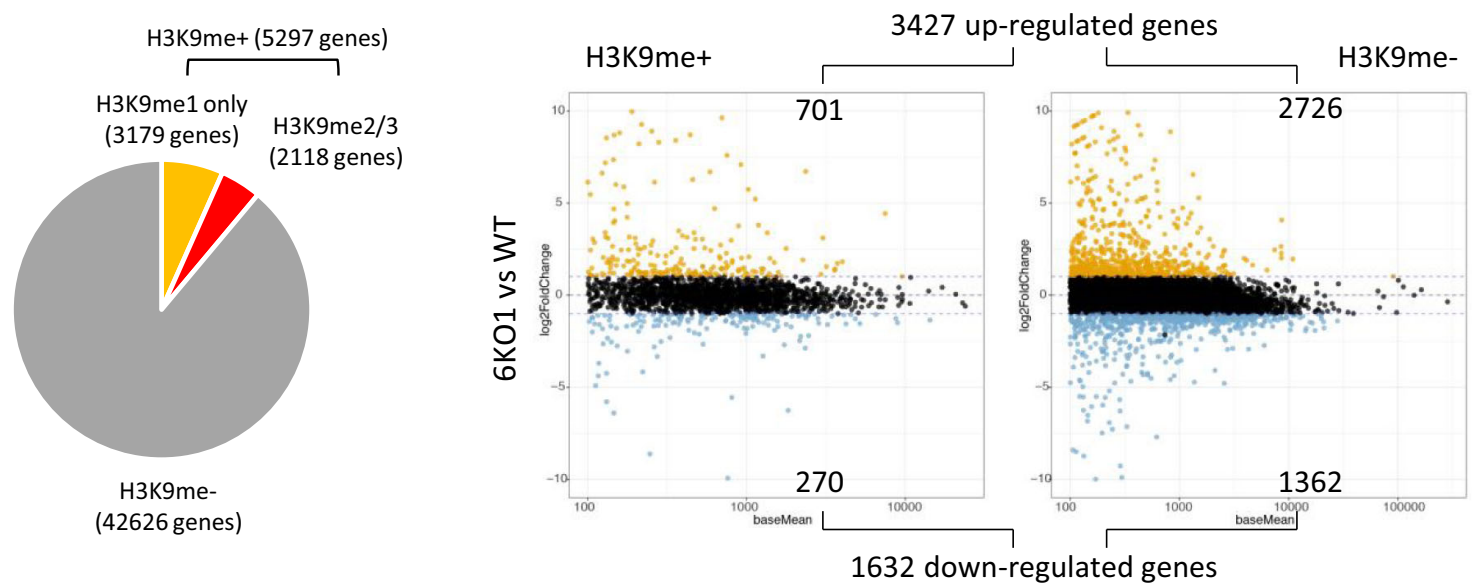

of the upregulated genes in the $6 \mathrm{KO} 1$ cells (701 of 3427 genes) (Fig. 5c) or $6 \mathrm{KO} 2$ cells (508 of 2517 genes), indicating an increased response of $\mathrm{H} 3 \mathrm{~K} 9 \mathrm{me}$-positive genes to loss of $\mathrm{H} 3 \mathrm{~K} 9$ methylation. By contrast, $80 \%$ of the genes derepressed in the $6 \mathrm{KO} 1$ mutants (2726 of 3427 genes) do not have $\mathrm{H} 3 \mathrm{~K} 9$ methylation at their promoters in WT cells. While some of these $\mathrm{H} 3 \mathrm{~K} 9$ me-negative genes might still be regulated by $\mathrm{H} 3 \mathrm{~K} 9$ methylation at distal sites, such as enhancers, this result suggests that the majority of the genes dysregulated in the compound H3K9 KMT mutants are not direct targets for the H3K9 KMT systems. Instead, we propose that the failure of repeat repression and the induction of DNA damage and stress signaling pathways in the compound $\mathrm{H} 3 \mathrm{~K} 9 \mathrm{KMT}$ mutants leads to widespread changes in gene expression. 
Fig. 5 Dysregulation of H3K9me-positive vs H3K9me-negative genes in compound H3K9 KMT-mutant MEF cells. a Differential expression of genes with H3K9 methylated promoter regions. Violin plots representing the distribution of gene expression levels in WT and compound H3K9 KMT mutants, as determined by RNA sequencing (RNA-seq, log scale). Red dots indicate mean expression level. The genes are grouped according to H3K9 methylation status over their promoter region, as determined by chromatin immunoprecipitation sequencing (ChIP-seq) in WT MEFs. Promoters are defined as within $+/-1 \mathrm{kilobase}$ ( $\mathrm{kb}$ ) from the transcription start site (TSS). From left to right, genes with promoters lacking any H3K9 methylation peak (H3K9me0, 42626 genes), followed by genes with promoters positive for H3K9me1 (3179 genes), H3K9me2 (270 genes), or H3K9me3 (462 genes). It is important to note that this analysis does not include genes with promoter regions that show enrichment for multiple H3K9 methylation states (as summarized in Supplementary Fig. 4B). b Genome browser tracks showing H3K9me1, H3K9me2, or H3K9me3 enrichment (log2 fold change, red) over example gene promoters, as determined by ChIP-seq in WT MEFs. From left to right, Bardet-Biedl syndrome 9 (Bbs9, H3K9me1-positive), Triggering receptor expressed on myeloid cells 4 (Trem/4, H3K9me2-positive) and Zinc finger protein 936 (Zfp936, H3K9me3-positive). Bottom shows RNA expression (RNA-seq, blue) in WT and compound H3K9 KMT mutants. Each browser track represents a $2 \mathrm{~kb}$ genomic interval surrounding the TSS. c Pie chart of genes classified by H3K9 methylation status at their promoter regions (left). Right, MA plots depicting changes in gene expression (RNA-seq) in 6KO1 cells relative to WT, shown for genes with (H3K9me +) or without (H3K9me-) H3K9 promoter methylation. Black dots represent genes whose changes in expression are not statistically significant. Yellow dots represent upregulated genes and blue dots downregulated genes. The total number of genes upregulated in $6 \mathrm{KO} 1$ cells is indicated above the plots, and the number of genes downregulated is shown below. The numbers of $\mathrm{H} 3 \mathrm{~K} 9 \mathrm{me}+$ and $\mathrm{H} 3 \mathrm{~K} 9 \mathrm{me}-\mathrm{up}$ - or downregulated genes are indicated on the corresponding sector of the MA plots. For graphical representation, only genes with base mean $>100$ are shown on the plots.

Partial dispersion of heterochromatic foci in Eset/Suv39h mutant MEF cells. We visualized heterochromatin organization in the series of compound H3K9 KMT-mutant MEF cells by FISH for MSR sequences (Fig. 6a). As described above (Fig. 1), Eset1, Eset2, or Eset1/Eset2 double-mutant cells display a nuclear clustering of MSR sequences that is indistinguishable from WT cells. MSR clustering is also maintained in E1E2 G9Gl deficient cells, although they display an increased number of smaller heterochromatin foci. By contrast, cells with a compound deficiency of Eset1 and Suv39h1/Suv39h2 enzymes (E1 S1S2) show a partial dispersion of heterochromatin, with a significant fraction of the cells (37\%) displaying elongated or generally irregular MSR foci (Fig. 6a). We also stained these cells with antibodies for $\mathrm{H} 3 \mathrm{~K} 9 \mathrm{me} 3$ and the heterochromatin protein HP1a (Supplementary Fig. 5A, B). Both $\mathrm{H} 3 \mathrm{~K} 9 \mathrm{me} 3$ and HP1a are completely dispersed in the nuclei of the E1 S1S2 mutant cells. This is not detected in compound H3K9 KMT mutants with functional Suv39h enzymes, even when all of the other four H3K9 KMT are inactivated (E1E2 G9Gl). We verified the specificity of these observations by analyzing the nuclear distribution of Hmga1, a protein that binds AT-rich sequences within pericentric chromatin $^{25,26}$. In all compound H3K9 KMT mutants, Hmgal signals remain intact and overlap with the DAPI-dense regions (Supplementary Fig. 5C).

To further investigate nuclear organization in the H3K9 KMT mutants, we visualized the integrity of the nuclear lamina by immunofluorescence detection of Lamin B (Fig. 6b). As we observed for Eset1/Eset2 double-null cells, all compound $\mathrm{H} 3 \mathrm{~K} 9$ KMT mutants lacking Eset1 function displayed multiple micronuclei and deformation of the nuclear lamina/envelope, in agreement with these cells expressing markers of DNA damage (see Supplementary Fig. 1C). We also analyzed the distribution of Fibrillarin, a protein-labeling nucleoli, and did not observe significant differences between the various compound $\mathrm{H} 3 \mathrm{~K} 9$ KMT mutants (Fig. 6c). These data suggest that the global organization of subnuclear compartments is not drastically altered in the H3K9 KMT mutants. Together, these observations confirmed that the spatial organization of heterochromatin is mostly dependent on Suv39h enzymes, although an additional inactivation of Eset (and probable defects in the nuclear lamina) in a Suv39h-deficient background is required for a partial breakdown of heterochromatin clustering, as previously reported $^{13}$.

Full collapse of heterochromatin in 6KO H3K9 KMT MEF cells. We next analyzed heterochromatin organization in MEF cells lacking all 6 KMT. Strikingly, FISH staining for MSR sequences revealed a complete collapse of heterochromatin foci in both $6 \mathrm{KO} 1$ and $6 \mathrm{KO} 2$ mutants (Fig. 6a). While not fully penetrant, this phenotype was observed in the majority of nuclei of both $6 \mathrm{KO} 1(63 \%)$ and $6 \mathrm{KO} 2(52 \%)$ cells. As for the E1 S1S2 cells, H3K9me3 and HP1a were also completely dispersed (Supplementary Fig. 5A, B). Hmgal remained enriched over DAPI-bright regions, even though these DAPI signals displayed an aberrant and non-clustered organization (Supplementary Fig. 5C). Since Hmgal was shown to also contribute to the integrity of heterochromatin foci ${ }^{26}$, its retention at the A/T-rich MSR-repeat sequences might explain, at least in part, the incomplete penetrance of heterochromatin dispersion in the $6 \mathrm{KO} \mathrm{MEF}$ cells.

Staining for Lamin B recapitulated defects in the nuclear lamina/envelope, as observed in all H3K9 KMT mutants lacking Eset 1 function, although the proportion of cells with micronuclei and the size of the micronuclei appeared increased in $6 \mathrm{KO} 1$ and $6 \mathrm{KO} 2$ cells (Fig. 6b). Fibrillarin staining indicated that the nucleoli structure was not significantly altered (Fig. 6c). Thus, complete loss of $\mathrm{H} 3 \mathrm{~K} 9$ methylation breaks heterochromatin clustering and reveals a definite function for $\mathrm{H} 3 \mathrm{~K} 9$ methylation in maintaining heterochromatin organization and genome integrity.

6KO H3K9 KMT MEF cells have lost electron-dense heterochromatin. We analyzed this collapse of heterochromatin at higher resolution by performing transmission electron microscopy (TEM) in the various H3K9 KMT mutants (Fig. 7). In WT (E1c) cells, heterochromatin is readily visualized as small clusters of electron-dense material, often localized close to the nuclear envelope (Fig. 7, white arrows), while larger electron-dense regions correspond to nucleoli. Eset1 (E1), Eset2 (E2), and Eset1/ Eset2 (E1E2) double mutants display a similar distribution of electron-dense heterochromatin as compared to wild type, with $>80 \%$ of cells displaying clearly defined heterochromatin clusters. Compound H3K9 KMT mutants lacking Eset1 and Suv39h/Suv39h2 (E1 S1S2) and, to some extent, Eset1/Eset2, G9a/ Glp (E1E2 G9Gl) show reduced electron density or lower number of foci, but maintain the distinction between heterochromatin and euchromatin. Strikingly, this is not the case for $6 \mathrm{KO} 1$ and $6 \mathrm{KO} 2 \mathrm{MEF}$ cells: in half of these cells analyzed by TEM, no heterochromatin clusters could be detected, although nucleoli are preserved (Fig. 7) and a further fraction of the $6 \mathrm{KO} 1$ and $6 \mathrm{KO} 2$ cells displayed markedly reduced electron density and only very faint foci. Around $81 \%(6 K O 1, n=26)$ or $78 \%(6 K O 2, n=14)$ of the cells have lost the clear distinction of electron-dense heterochromatin. These data provide compelling evidence for the crucial requirement of $\mathrm{H} 3 \mathrm{~K} 9$ methylation in maintaining heterochromatin organization. 
a
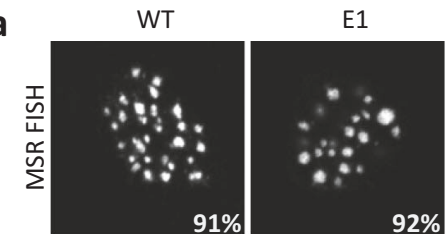

E1S1S2

$6 \mathrm{KO} 1$
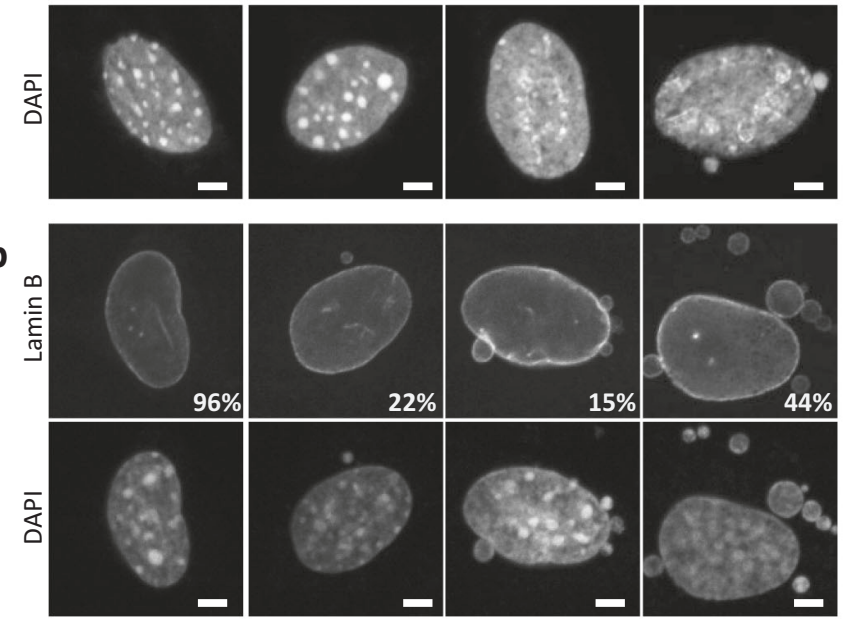

C
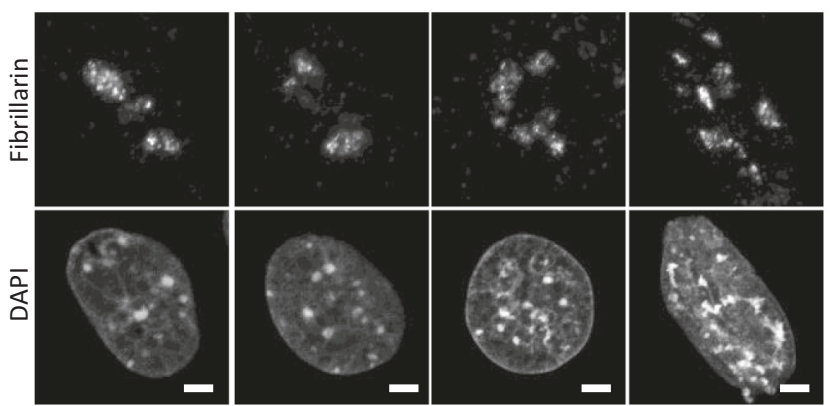

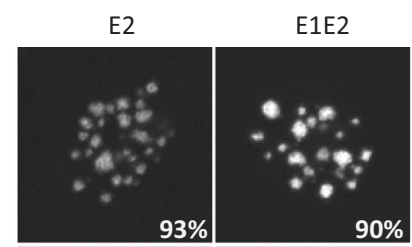

E1E2 G9GI

$6 \mathrm{KO} 2$
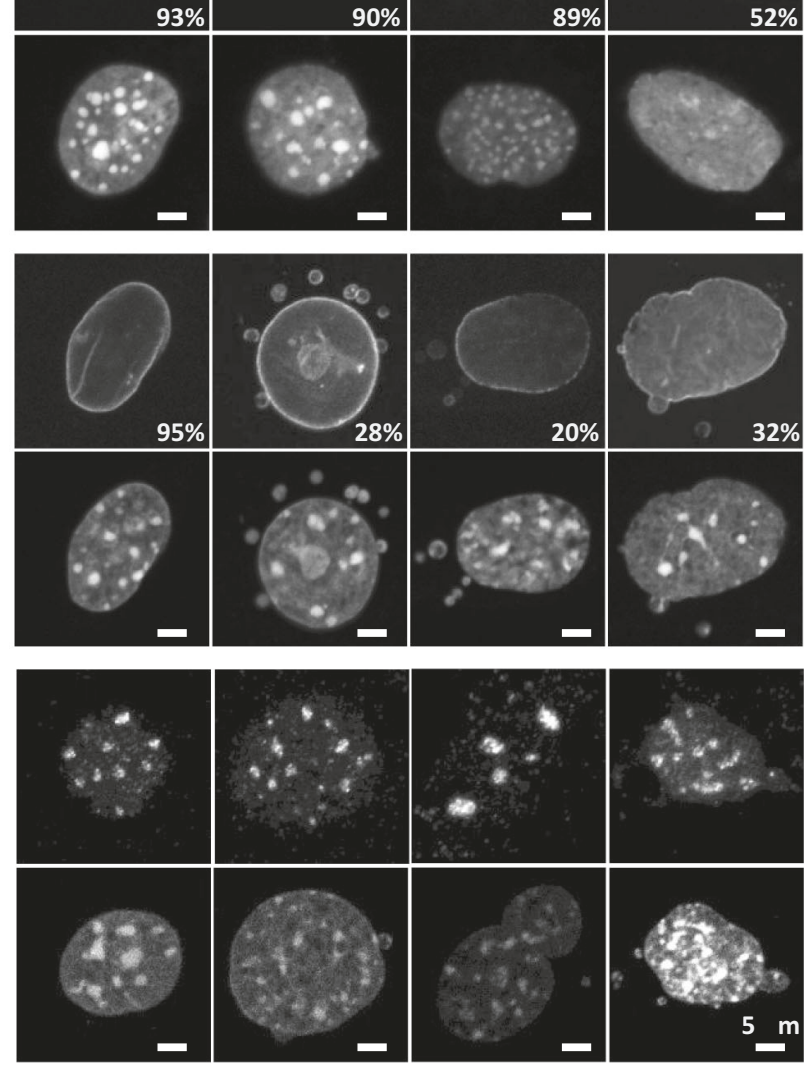

Fig. 6 Collapse of heterochromatin in compound H3K9 KMT-mutant MEF cells. a DNA FISH for major satellite repeats (MSR) in control and compound H3K9 KMT mutant. Percentages indicate cells that display the shown pattern. N=501 cells examined for WT, 303 for E1, 352 for E1 S1S2, 304 for 6KO1, 308 for E2, 311 for E1E2, 301 for E1E2 G9GI, and 309 for 6 KO2 over three independent experiments. For each image, lower panels show DAPI counterstaining of the same nuclei. $\mathbf{b}$ Immunofluorescence for Lamin B, marking the nuclear lamina. Percentages indicate cells that display the shown pattern. $N=680$ cells examined for WT, 785 for E1, 302 for E1 S1S2, 305 for 6KO1, 573 for E2, 499 for E1E2, 558 for E1E2 G9Gl, and 427 for 6KO2 over three independent experiments. For each image, lower panels show DAPI counterstaining of the same nuclei. c Immunofluorescence for Fibrillarin, marking nucleoli.

Representative images ( $N=100$ cells) are shown. For each image, lower panels show DAPI counterstaining of the same nuclei. Scale bars represent $5 \mu$ m.

\section{Discussion}

Heterochromatin forms distinct chromatin domains that can be visualized by DAPI staining of A/T-rich major satellite repeats in mouse cells. Suv39h enzymes are the central KMT for heterochromatic $\mathrm{H} 3 \mathrm{~K} 9 \mathrm{me} 3$. However, while heterochromatin is weakened in Suv39h double-null cells, its overall organization and clustering remain largely intact. Many mechanisms could contribute to this heterochromatin stability independently of $\mathrm{H} 3 \mathrm{~K} 9$ methylation, including specific chromosome conformations, such as the Rabl configuration or anchoring to the nuclear membrane or other subnuclear structures $3,27,28$. For example, the threedimensional organization of chromosomes in S. pombe involves both H3K9 methylation, which controls chromatin compaction near centromeres, and cohesin-mediated formation of globular domains along chromosome arms ${ }^{27}$.

Another possibility is that other silencing systems can partially compensate for the loss of Suv39h enzyme function, such as Polycomb Repressive Complexes (PRC) and/or DNA methylation. We indeed observed a moderate increase in H3K27me3 levels in 6KO MEF cells chromatin, while global DNA methylation was only slightly decreased over the examined number of cell divisions. Recruitment of Polycomb-mediated H3K27me3 at heterochromatin has already been observed in other contexts where H3K9 methylation is reduced $5,29,30$.

The only minor decline of overall $5 \mathrm{mC}$ levels seems incoherent with the well-documented interdependence between $\mathrm{H} 3 \mathrm{~K} 9$ chromatin and CpG DNA methylation ${ }^{31}$. However, in lineagecommitted cells, reduction of H3K9 methylation does not cause a global loss of DNA methylation at heterochromatin, as, for example, Suv39h double-null MEF cells keep high levels of $5 \mathrm{mC}$ at many repeat elements ${ }^{32}$. Maintenance of DNA methylation by Dnmtl is indeed not strictly dependent on recruitment to H3K9 methylated nucleosomes ${ }^{33,34}$. Further, $\mathrm{CpG}$ methylation is not restricted to heterochromatin regions, but also abundant on transcribed gene bodies, where it is established by H3K36me3mediated recruitment of Dnmt $3 b^{35}$. Determining whether and how these other silencing pathways are redirected or adjusted in the H3K9 KMT mutants would require genome-wide ChIP mapping of $\mathrm{H} 3 \mathrm{~K} 27 \mathrm{me} 3$ and genome-wide bisulfite sequencing for $5 \mathrm{mC}$.

We observed a high incidence of micronuclei and aberrant shape of the nuclear lamina/envelope in compound H3K9 KMTmutant cells that correlated with transcriptional signatures indicating the activation of DNA damage pathways. This is in 


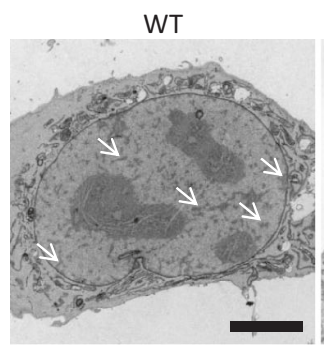

$19 / 27(89 \%)$

E2

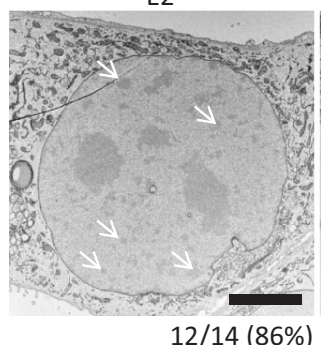

$12 / 14(86 \%)$

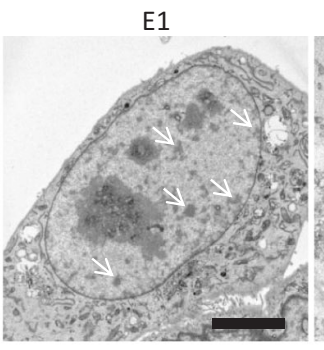

$15 / 15(100 \%)$

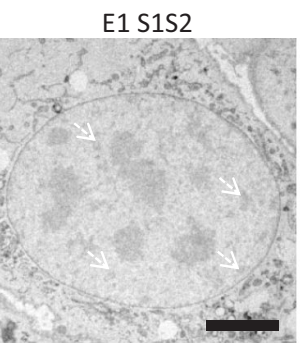

$10 / 15(67 \%)$

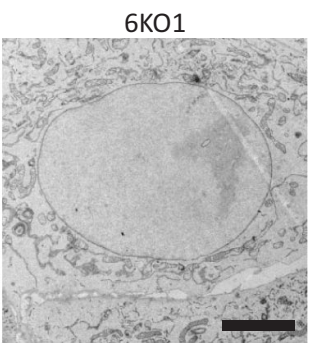

$21 / 26(81 \%)$

E1E2 G9GI
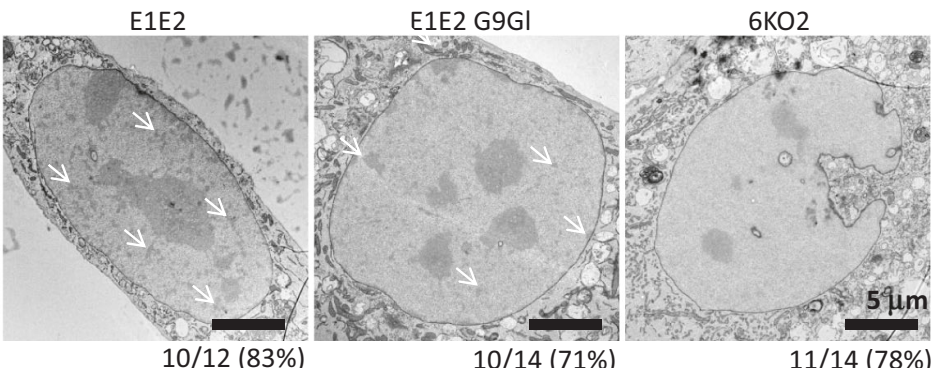

Fig. 7 Loss of electron-dense heterochromatin in MEF cells lacking all 6 SET-domain H3K9 KMT. Transmission electron microscopy (TEM) images of nuclei from compound H3K9 KMT mutants. Each panel shows a representative TEM image of a nucleus of the indicated H3K9 KMT mutant. White arrows point to electron-dense heterochromatin clusters. The total number of cells analyzed and the percentage of cells displaying the shown pattern are indicated below each image. Scale bars represent $5 \mu \mathrm{m}$.

agreement with the known roles of heterochromatin in the maintenance of genome integrity that include protecting cells from replication stress, chromosome segregation errors, or aberrant recombination of repeats, as it was reviewed recently ${ }^{3}$. Interestingly, these defects were most pronounced in $\mathrm{H} 3 \mathrm{~K} 9 \mathrm{KMT}$ mutants where Eset1 is inactivated and they also occurred in chromatin that maintains high levels of $\mathrm{H} 3 \mathrm{~K} 9$ methylation. These data suggest that in addition to the H3K9 histone, Eset1 has other non-histone substrates, some of which could function in the control of DNA damage and in correcting replication errors. Increased DNA damage was reported following Eset 1 deletion in mouse oocytes or in intestinal epithelium ${ }^{36,37}$.

The $6 \mathrm{KO} \mathrm{H} 3 \mathrm{~K} 9 \mathrm{KMT}$ display a collapse of heterochromatin foci and loss of electron-dense heterochromatin. This reveals an essential function for $\mathrm{H} 3 \mathrm{~K} 9$ methylation in maintaining heterochromatin as a separate nuclear domain. Our data also show a larger degree of cooperation among the 6 SET-domain H3K9 KMT to secure heterochromatin integrity. Conversely, this seems to exclude a contribution to $\mathrm{H} 3 \mathrm{~K} 9$ methylation and heterochromatin organization of other KMT systems, such as split SETdomain enzymes or "seven-beta-strand" (7 $\beta s) \mathrm{KMT}^{38}$. The described dispersion of heterochromatin in $\operatorname{Prdm} 3 / \operatorname{Prdm} 16$ shRNA MEF mutants ${ }^{13}$ is probably related to concurrent secondary defects in the structuring of the nuclear lamina and not caused by a reduction of $\mathrm{H} 3 \mathrm{~K} 9$ methylation in nuclear chromatin.

H3K9 KMT mutants of any distinct gene pair of either Suv39h1/Suv39h2, Eset1/Eset2, or G9a/Glp maintain heterochromatin structure, indicating that functional redundancy between these SET-domain H3K9 KMT ensures the robustness of heterochromatin organization. Importantly, the contribution of each of these six SET-domain H3K9 KMT may be different in other cell types. For example, Suv39h1-null T cells start to disintegrate heterochromatic foci, even though the other $5 \mathrm{H} 3 \mathrm{~K} 9$ KMT remain functional ${ }^{39}$. Similarly, Eset $1 /$ Suv39h compound mutant hepatocytes also have severely reduced EM-dense heterochromatin ${ }^{40}$.

What is the mechanism for the collapse of heterochromatin in the compound H3K9 KMT mutants? Chromatin that is deficient for all $\mathrm{H} 3 \mathrm{~K} 9$ methylation states will not provide binding for HP1 proteins and may have lost the physicochemical properties to engage in phase separation ${ }^{3,41,42}$. While the dispersion of HP1 binding is not sufficient to break heterochromatin ${ }^{11}$, other H3K9me2 or H3K9me1-binding proteins might substitute for HP1 in Suv39h-deficient cells, but not in compound H3K9 KMTmutant cells that lack all H3K9 methylation states. Possibly, some of these putative $\mathrm{H} 3 \mathrm{~K} 9$-methyl binders could also assist in phase separation, and thus increase heterochromatin stability. Alternatively, there could also be non-histone substrates for the distinct SET-domain H3K9 KMT, particularly for Eset, that are needed for heterochromatin organization but fail to become methylated in the $6 K O$ MEF cells.

Several proteins were identified as candidate anchoring factors for heterochromatin. In mouse cells, Lamin B receptor (LBR) or Lamin A/C tether heterochromatin to the inner nuclear membrane in a cell-type-specific manner, but the interaction is likely indirect and adaptor proteins remain to be identified ${ }^{43}$. In C. elegans, the chromodomain containing protein CEC-4 binds methylated $\mathrm{H} 3 \mathrm{~K} 9$ and is required for recruitment of heterochromatin at the nuclear periphery, but dispensable for its silencing ${ }^{44}$. While no clear homolog of CEC-4 is found in mammals, other chromodomain proteins might function in a similar way.

Answering these questions will require future studies and would be facilitated if conditions for compound H3K9 KMTmutant cells can be generated that allow the analysis of collapsed heterochromatin not only at its onset (this study) but also for more prolonged cell divisions. In summary, our work provides compelling evidence for a crucial function of $\mathrm{H} 3 \mathrm{~K} 9$ methylation in heterochromatin organization.

\section{Methods}

Eset1-conditional MEF. Eset1-conditional MEFs were described previously ${ }^{6}$. To optimize deletion efficiency, we sorted single cells in 96-well plates and selected for cell clones with high Cre activity (clone Eset25, referred to as E1c). After CRISPR Cas9-mediated gene disruption (see below), we further re-introduced a construct expressing tamoxifen-inducible Cre together with a puromycin resistance gene (pCAGGS-CreERT2-EGFP-IRES-PURO), and maintained the cell lines under constant puromycin selection. Cre-mediated deletion of exons 15 and 16 of Eset 1 was induced by growing the cells in a medium containing $1 \mu \mathrm{M}(\mathrm{Z})-4$ - 
hydroxytamoxifen (4-OHT, Sigma Aldrich, H7904) for 2 days, followed by 2 days in normal medium, to minimize potential side effects of prolonged growth in presence of tamoxifen. Deletion efficiency was assessed by RT-qPCR with primers specific for the deleted exons, or by western blot for the Esetl protein. We refer to E1c as WT.

CRISPR/Cas9 mutations. CRISPR/Cas9-mediated gene disruptions were performed by transfection of chimeric sgRNA/Cas 9 expression vectors ${ }^{45}$. For each SET-domain KMT target gene, two unique guide RNAs were designed using online tools (CRISPR.mit.edu) and subsequently cloned in the chimeric sgRNA/Cas9 expression vectors pSpCas9(BB)-2A-Puro (pX459) or pSpCas9(BB) (pX330), both from Addgene. Cells were transfected with Xfect (Clontech) and selected for 2 days in puromycin-containing media. After selection, the efficiency of Cas9-mediated cleavage was evaluated by Surveyor assay ${ }^{45}$. Puromycin-resistant cells were further sorted to single cells in 96-well plates, and individual clones analyzed for the absence of functional protein by western blotting when specific antibodies were available, or for the absence of a functional copy of the gene by sequencing the relevant exons (for Eset2). The sequences of the guide RNAs used in this study are listed in Supplementary Table 1.

Gene pair mutations. For gene pair mutants, we transfected combinations of chimeric sgRNA/Cas9 expression vectors in E1c MEFs and selected mutant clones as described above. Conditional Eset1, Eset2-null mutants (E1cE2) were obtained by introducing a single guide RNA specific for Eset 2 into E1c MEFs. Clone A4 was selected after sequencing revealed a frameshift mutation within exon 7 of Eset2. G9a/Glp double mutants were generated by co-transfection of two sgRNA/Cas 9 vectors targeting $G 9 a$ and $G l p$. Clone $\mathrm{H} 7$ was selected after western blot analysis confirmed the absence of G9a or Glp proteins. Suv39h-deficient cells were generated by co-transfection of two sgRNA/Cas9 vectors targeting Suv39h1 and Suv39h2 in E1c MEFs. Clone B1 was selected after western blot analysis confirmed the absence of Suv39h1 or Suv39h2 proteins.

Compound SET-domain H3K9 KMT mutants. Compound H3K9 KMT mutants were generated by successive rounds of sgRNA/Cas 9 transfection and selection of relevant clones. The nomenclature of the clones refers to null mutation of SETdomain KMT genes and is indicated as E1 (Eset1-null), E2 (Eset2-null), G9 (G9anull), Gl (Glp-null,) S1 (Suv39h1-null), and S2 (Suv39h2-null).

Series 1 clones were generated from Eset1-conditional MEF cell, after the first round of selection for high Cre recombinase activity resulting in the isolation of the Eset25 clone, referred to as E1c (see above). E1c cells were transfected with two sgRNA/Cas9 vectors targeting Suv39h1 and Suv39h2 and clone B1 (E1c S1S2) was selected as described above. This clone was further co-transfected with vectors targeting Eset2, G9a and Glp, and clone B1A6 (E1cE2 S1S2 G9Gl) was selected after western blotting (G9a, Glp) and exon sequencing (Eset2).

Series 2 was generated from clone A4 (E1cE2) described above. We first cotransfected vectors targeting $G 9 a$ and $G l p$ and selected clone E2 (E1cE2 G9Gl), which was subsequently transfected with vectors targeting Suv39h1 and Suv39h2 to generate clone E2F4 (E1cE2 G9Gl S1S2). After inducing Eset1 deletion, cells lacking all $6 \mathrm{H} 3 \mathrm{~K} 9 \mathrm{KMTs}$ are referred to as $6 \mathrm{KO} 1$ and $6 \mathrm{KO} 2$.

Cellular extracts and western blot analysis. Nuclear extracts from 10 million cells were prepared by cell lysis for $30 \mathrm{~min}$ at $4{ }^{\circ} \mathrm{C}$ in hypotonic buffer $(10 \mathrm{mM}$ HEPES pH 7.9, $5 \mathrm{mM} \mathrm{MgCl}_{2}, 0.25 \mathrm{M}$ sucrose, $0.1 \% \mathrm{NP}-40$ ) and protease inhibitors (Roche). Nuclei were isolated by centrifugation and washed in lysis buffer. Acid extraction of histones ${ }^{46}$ was performed in $0.4 \mathrm{~N} \mathrm{HCl}$ at $4{ }^{\circ} \mathrm{C}$ overnight, followed by centrifugation to discard insoluble material and by neutralization with $\mathrm{NaOH}$. For whole-cell extracts, cell pellets from 5 million cells were washed in PBS and lysed in RIPA buffer. SDS-PAGE analysis was conducted according to standard procedures, with antibodies against Gapdh (Santa Cruz, sc-3233, 1:5000 dilution), Setdb1 (Thermo Fisher, MA5-15722, 1:1000 dilution), G9a (Abcam, ab185050, 1:2500 dilution), Glp (Abcam, ab41969, 1:2500 dilution), Suv39h1 (Cell signaling, 8729, 1:2500 dilution), Suv39h2 (LSBio, LS-C116360, 1:2500 dilution), total H3 (Abcam, ab1791, 1:40000 dilution), H3K9mel (Active Motif, 39681, 1:2500 dilution), H3K9me2 (Jenuwein Laboratory, \#4677, 1:5000 dilution), H3K9me3 (Abcam, ab8898, 1:20000 dilution), H3 pan-acetyl (Millipore, 06-599, 1:1000 dilution), H4 pan-acetyl (Millipore, 06-866, 1:2500 dilution), H3K4me3 (Diagenode, C1540003, 1:1000 dilution), $\alpha$-tubulin (Abcam, ab4074, 1:1000 dilution), or $\gamma \mathrm{H} 2 \mathrm{~A} . \mathrm{X}$ (Millipore 05-636, 1:750 dilution). As a positive control for $\gamma$ H2A.X detection, E1c MEFs were treated with Etoposide (Sigma E383), either for $3 \mathrm{~h}$ at $12.5 \mu \mathrm{M}$, or for $24 \mathrm{~h}$ at $1 \mu \mathrm{M}$.

Histone extraction and nanoLC-MS analysis. After acid extraction of histones, histones samples were separated on 16\% SDS Tris Glycine Wedge gels (Thermo Fisher) followed by colloidal coomassie staining (Instant Blue, Expedeon). Histone bands were excised and histone propionylation and subsequent protein digestion using trypsin (Promega) were performed as described ${ }^{47}$, with minor modifications. In-gel propionylation prior to trypsin digestion was conducted two times employing the method " $\mathrm{H}-42 \mathrm{x}$ " 47 followed by the hydroxylamine-mediated reversal of overpropionylation. Peptide samples were desalted using STAGE tips ${ }^{48}$ and analyzed using LC-MS as previously described ${ }^{49}$ with minor modifications.
Samples were separated within 60 min with a linear gradient from $2 \%$ buffer B ( $80 \%$ acetonitril in $0.1 \%$ formic acid) to $60 \%$ buffer B at a flow rate of $250 \mathrm{nl} / \mathrm{min}$ and this was followed by a washout step ( $80 \%$ buffer B, $5 \mathrm{~min}, 500 \mathrm{nl} / \mathrm{min})$ and reequilibration ( $2 \%$ buffer B, $5 \mathrm{~min}, 500 \mathrm{nl} / \mathrm{min}$ ). Mass spectrometry was carried out using the "sensitive method" (data-dependent acquisition) on Orbitrap QExactive Plus (Thermo Fisher Scientific) ${ }^{50}$

Mass spectrometry raw data were analyzed using Peaks Studio version 8.5 (Bioinformatics Solution Inc.). MS raw files were searched using the Peaks de novo search algorithm and Peaks DB search against Mus musculus (mouse) UniprotKB protein sequence database (December 2015). Trypsin was selected as a cleaving enzyme, while a maximum of four missed cleavages, a minimum of six amino acids per peptide, $10 \mathrm{ppm}$ MS peptide ion mass tolerance, and 0.2 Da MS/MS tolerance were allowed. Carbamidomethylation of cysteine was set as a fixed modification, whereas the following modifications were set as variable modifications: acetylation at the protein $\mathrm{N}$ terminus and at lysine residues, deamidation of asparagine or glutamine, oxidation of methionine, propionylation of lysine and monomethylated lysine residues, mono-methylation of lysine and arginine residues, dimethylation of lysine and arginine residues, trimethylation of lysine residues, and phosphorylation of serine, threonine, and tyrosine. Maximum (allowed) variable PTMs per peptide were set to 4 . The peptide and protein false discovery rate (FDR) was set to a maximum of 0.01 using the decoy-fusion database search strategy implemented in Peaks Studio.

Histone peptide intensities were exported and the sum of all peptide intensities (or features), belonging to each particular histone PTM (hPTM) mark, was calculated for histone $\mathrm{H} 3$ and histone $\mathrm{H} 4$ in each individual biological sample. This was followed by an inter-sample normalization to adjust the total intensity of all hPTMs to the same level in each biological replicate in each condition. To achieve this normalization, the summed peptide intensity of each particular hPTM was divided by the total intensity of a non-modified histone peptide, a histone peptide that is consistently identified and bears no hPTM mark throughout the whole experiment. Peptide "YRPGTVALR" and peptide "VFLENVIR" were used for normalizing mouse histone $\mathrm{H} 3$ (sequence: 41-49) and mouse histone $\mathrm{H} 4$ (sequence: 60-67), respectively.

Data were plotted using GraphPad Prism version 8.3.0.

DNA methylation analysis. Genomic DNA was purified by phenol/chloroform extraction and ethanol precipitation according to standard procedures. DNA dot blot for $5 \mathrm{mC}$ was performed according to standard procedures ${ }^{51}$. DNA was spotted on nitrocellulose (Biorad) in $2 \times$ SSC and UV-cross-linked at $254 \mathrm{~nm}, 120 \mathrm{~mJ} / \mathrm{cm}^{2}$ Membranes were probed with anti-5-methylcytosine antibody (Zymo research, clone 10G4, diluted 1:3000 in 3\% BSA/PBST). For MS analysis of $5 \mathrm{mC}$ and $5 \mathrm{hmC}$ levels, genomic DNA was digested to nucleosides using Nucleoside Digestion mix (NEB, M0649S). Targeted nucleoside quantification by LC-MS was carried out using an Agilent 1290 Infinity II UHPLC inline with an Agilent 6495 QQQ-MS operating in MRM mode. MRM settings were optimized separately for unmodified nucleosides using pure standards and adapted for methylated nucleosides. LC separation was on a Waters T3 column $(100 \times 2.1 \mathrm{~mm}, 1.8-\mu \mathrm{m}$ particles $)$ using a solvent gradient of $100 \%$ buffer A ( $0.1 \%$ formic acid in water) to $90 \%$ buffer B (50:50 acetonitrile:methanol). Flow rate was $400 \mu \mathrm{L} / \mathrm{min}$. Autosampler temperature was $4{ }^{\circ} \mathrm{C}$, and the injection volume was $2 \mu \mathrm{L}$. Data processing was performed using Agilent MassHunter Software (version B.08)

Indirect immunofluorescence and DNA FISH. Cells were grown on glass coverslips, washed with PBS, fixed at room temperature with $2 \%$ PFA for $10 \mathrm{~min}$, and permeabilized with $0.5 \%$ Triton X-100 for 5 min followed by PBS washes. Immunofluorescence was performed with antibodies against $\mathrm{H} 3 \mathrm{~K} 9 \mathrm{me} 3$ (Jenuwein Laboratory, \#1926, 1:1000 dilution in 10\% FBS/PBS), Lamin B (Santa Cruz, sc6217, 1:200 dilution), HP1 $\alpha$ (Millipore, 05-689, 1:500 dilution), Fibrillarin (Thermo Fisher, MA3-16771, 1:500 dilution), and Hmgal (Abcam, ab129153, 1:500 dilution) for $1 \mathrm{~h}$ at room temperature. For DNA FISH, cells were dehydrated in ethanol. Denaturation at $80^{\circ} \mathrm{C}$ for $5 \mathrm{~min}$ and hybridization at $37^{\circ} \mathrm{C}$ for $1 \mathrm{~h}$ were performed in hybridization solution $(2 \times \mathrm{SSC} \mathrm{pH} 7,50 \mathrm{mM}$ sodium phosphate $\mathrm{pH}$ $7,60 \%$ formamide) with a mix of 4 LNA probes ( $3 \mathrm{nM}$ each). Probe sequences are listed in Supplementary Table 1. Image acquisition was performed on an LSM 780 microscope (Zeiss) using ZEN2 black edition software (Zeiss).

RNA sequencing. Total RNA was extracted with Trizol (Invitrogen), and the remaining DNA was digested with Turbo DNase (Ambion), followed by cleanup with RNeasy MinElute Cleanup kit (Qiagen, 74204). Total RNA libraries were prepared using the TruSeq Stranded Total RNA Library Prep Gold (Illumina, 20020598). Paired-end, $75 \mathrm{bp}$ reads were generated with Illumina HiSeq3000 sequencer with a depth of 50 million reads per sample. Three biological replicates were sequenced per cell line.

RNA-seq analysis. Unprocessed reads were first quality and adapter trimmed using cutadapt ${ }^{52}$ version 1.8 .1 using a quality threshold of 20 . Reads were then aligned to the GRCm38 genome from Ensembl using STAR $^{53}$ version 2.5.2b, with settings as recommended by TEtranscripts ${ }^{54}$. Both repeats and genes were then quantified using TEtranscripts version 2.0.3 and differentially expressed repeat 
elements and genes determined using DESeq2 $2^{55}$. For analysis of repeat element expression, library size factors as computed from the gene expression data were used rather than recomputing these using the expression metrics of the repeat elements themselves. BigWig files using in visualization tracks were constructed using deepTools ${ }^{56}$. For pathway analyses, normalized counts as computed by DESeq2, as well as fold changes and adjusted P values, were loaded into Ingenuity Pathway Analysis and the results subsequently plotted in R.

For visualization of coverage over repeat regions (Fig. 3c) reads where aligned to the repeat sequence extracted from Repbase ${ }^{57}$ using bwa mem ${ }^{58}$ version 0.7.17 with default settings, bigWig tracks were constructed using deepTools and IGV ${ }^{59}$.

ATAC sequencing. For ATAC library preparation ${ }^{60}, 50,000$ cells were lysed in cold lysis buffer (10 mM Tris-HCl pH 7.4, $10 \mathrm{mM} \mathrm{NaCl}, 3 \mathrm{mM} \mathrm{MgCl}_{2}, 0.1 \% \mathrm{v} / \mathrm{v}$ Igepal CA-630) and centrifuged at $4^{\circ} \mathrm{C}$ for $10 \mathrm{~min}$ at $500 \mathrm{~g}$. The pellet was resuspended in a transposition reaction mixture provided by the Nextera DNA Library Prep kit (Illumina, 15028212) and incubated at $37^{\circ} \mathrm{C}$ for $30 \mathrm{~min}$. After transposition, the samples were purified with a Qiagen MinElute PCR purification kit (28604) according to the manufacturer's instructions. For library preparation, each sample was subjected to PCR amplification with a pair of barcoded primers provided by the Nextera Index kit (Illumina 15055289). The reaction was monitored by qPCR and further cycles were performed when necessary, based on the amplification plot. Paired-end, 75 bp reads were generated with Illumina HiSeq3000 sequencer with a depth of 50 million reads per sample. Two biological replicates were sequenced per cell line.

ATAC-seq analysis. Unprocessed reads were first quality and adapter trimmed using fastp ${ }^{61}$ version 0.19 .4 , using a mean window quality threshold of 3 and excluding low-complexity sequences. These were then aligned to the GRCm38 genome from Ensembl using STAR $^{53}$ version 2.6.0c allowing no spliced alignments and with other options as described by TEtranscript ${ }^{54}$. For extraction of the openchromatin subset of reads, deepTools ${ }^{56}$ version 3.1.2 was used with a fragment length threshold of 150 bases. Alignments were subsequently quantified across repeats using TEtranscripts version 2.0.3 and the repeatmasker file from UCSC as the source for repeat locations. For visualization, bigWig tracks were constructed using deepTools and IGV ${ }^{59}$. Differential accessibility was determined using DESeq $2^{55}$ version 1.20.0. Counts across gene bodies for genes showing unchanged expression in the RNA-seq data were used to determine sample-specific size factors, as other normalization methods would wash away global accessibility shifts. Pathway analysis was performed as with the RNA-seq data.

Chromatin immunoprecipitation sequencing (ChIP-seq). Ten million cells were trypsinized, washed with PBS, and cross-linked in $1 \%$ formaldehyde for $10 \mathrm{~min}$ at room temperature. After quenching with glycine $(0.125 \mathrm{M})$, cells were pelleted, washed with cold PBS, and lysed in $1 \%$ SDS, $10 \mathrm{mM}$ EDTA, $50 \mathrm{mM}$ Tris$\mathrm{HCl}, \mathrm{pH} 8$, and protease inhibitors (Roche). Lysates were sonicated (Covaris S220) to fragment sizes of 200-500 bp. For each ChIP, chromatin corresponding to $10 \mu \mathrm{g}$ of DNA was diluted in $1 \%$ Triton X-100, 2 mM EDTA, 167 $\mathrm{mM} \mathrm{NaCl}, 20 \mathrm{mM}$ Tris- $\mathrm{HCl}, \mathrm{pH} 8$, and protease inhibitors, and incubated overnight at $4{ }^{\circ} \mathrm{C}$ with $4 \mu \mathrm{g}$ of antibody against H3K9mel (Abcam, ab8896), H3K9me2 (Abcam, ab1220) or H3K9me3 (Abcam, ab8898), followed by $4 \mathrm{~h}$ at $4{ }^{\circ} \mathrm{C}$ with $25 \mu \mathrm{l}$ of Protein $\mathrm{G}$ dynabeads (Invitrogen). Beads were washed three times in low salt $(150 \mathrm{mM} \mathrm{NaCl})$ and once in high salt $(500 \mathrm{mM} \mathrm{NaCl})$ before elution in $1 \%$ SDS, $50 \mathrm{mM} \mathrm{NaHCO}_{3}$, RNAse A and Proteinase $\mathrm{K}$ treatment, and DNA purification with Qiagen MinElute PCR purification kit (28604).

Enrichment was validated by qPCR for control loci (Supplementary Fig. 3A: Major satellite repeats, L1MdA 5'UTR, IAP-EY3, Zinc finger protein 180 and Oct4 promoter as positive controls, as well as Actin B promoter as a negative control). Primer sequences are listed in Supplementary Table 1. Libraries were prepared with NEBNext Ultra II DNA Library Prep Kit for Illumina (NEB, E7645L). Paired-end, 100 bp reads were generated with Illumina NovaSeq 6000 with a depth of 100 million reads per sample. Two biological replicates were sequenced per ChIP as well as for input material.

ChiP-seq analysis. The quality of raw Fastq reads was checked using FastQC (http:// www.bioinformatics.babraham.ac.uk/projects/fastqc). Reads were aligned to $\mathrm{mm} 10$ (GRCm38) with bwa version 0.7.1. ${ }^{58}$. Peaks were called using histoneHMM ${ }^{62}$. Peaks were annotated using ChiPseeker software ${ }^{63}$ version 1.18.0. Peaks that are located within $+/-1 \mathrm{~kb}$ of the TSS were extracted for downstream analysis. Heatmaps and profiles were generated using deeptools version 3.5.056. Marks were aligned around the center of the peak (computeMatrix reference-point). Flanking regions were added to show the signal extending outside enriched regions. ComputeMatrix was executed with the following parameters:-skipZeros-binSize 10 -a 10000 -b 10000-referencePoint center. Heatmaps were generated using plotHeatmap. Coverage and normalization to the input were obtained using deepTools-3.5.0 bamCompare. FRiPs scores were obtained using deepTools/plotEnrichment. Violin plots represent the distribution of gene expression levels for each H3K9 methylation state. Normalized values of RNA-seq expression were obtained using DESeq2 version $1.26 .0^{55}$ on the count matrices output from snakePipes version 2.1.064. Red dots represent the mean expression level. Data visualization was performed using ggplot $2^{65}$ version 3.3.2. $P$ values comparing the means were calculated using the $t$ test.

Transmission electron microscopy (TEM) sample preparation and imaging Cells grown on Thermanox coverslips (Nunc) were prepared as described ${ }^{66}$ and adapted for cell monolayers. Cells were fixed with $2.5 \%$ glutaraldehyde and $2 \%$ paraformaldehyde (Electron Microscopy Sciences EMS, 16400 and 15700) in $0.1 \mathrm{M}$ Hepes buffer $\mathrm{pH} 7.4$ for $1 \mathrm{~h}$ at room temperature and then overnight at $4{ }^{\circ} \mathrm{C}$. After washes in cold $0.15 \mathrm{M}$ cacodylate buffer $\mathrm{pH} 7.4$ (Sigma Aldrich, CO250) the cells were post-fixed in 1.5\% potassium ferrocyanide (Sigma Aldrich, 60280) and 2\% osmium tetroxide (EMS, 19110) in $0.15 \mathrm{M}$ cacodylate buffer $\mathrm{pH} 7.4$ on ice for $30 \mathrm{~min}$. The cells were then washed with double-distilled water $(\mathrm{ddH} 2 \mathrm{O})$ and immersed in filtered $1 \%$ thiocarbohydrazide (TCH, EMS, 21900) solution for $10 \mathrm{~min}$. After this step, the cells were stained in $2 \%$ osmium tetroxide for $30 \mathrm{~min}$, rinsed again in $\mathrm{ddH}_{2} \mathrm{O}$, and stained with $1 \%$ uranyl acetate at $4{ }^{\circ} \mathrm{C}$ overnight. After washes with $\mathrm{ddH}_{2} \mathrm{O}$, cells were stained with Walton's lead aspartate (Sigma A9256 and EMS 17900) solution at $60^{\circ} \mathrm{C}$ for 30 min and dehydrated in a graded alcohol series. After flat-embedding in Epoxy resin (SERVA Electrophoresis GmbH 21045) samples were cured at $60^{\circ} \mathrm{C}$ for $12 \mathrm{~h}$. Coverslips were detached from the resin using repetitive freeze-thaw in liquid nitrogen. A region of interest containing cells was selected $(\sim 1 \times 1 \mathrm{~mm})$, cut, and mounted on an aluminum pin. For transmission electron microscopy (TEM), serial thin sections $(60 \mathrm{~nm})$ were cut using an ultramicrotome (Ultracut 7, Leica, Vienna) and imaged using a Spirit $120 \mathrm{kV}$ (FEI, now THF, Eindhoven) at magnifications between $1700 \times$ and $8200 \times$ equipped with a side-mounted camera (Veleta, EMSIS Gmbh).

\section{Data availability}

The proteomics datasets generated in this study are available at ProteomeXchange via the PRIDE partner repository with record number PXD018175. The UniProtKB database is accessible at https://ftp.uniprot.org/pub/databases/uniprot/previous_major_releases/ release-2015_12/knowledgebase/. A database filtered for mouse peptides is available as a FASTA file at ProteomeXchange, together with the datasets generated in this study. The RNA-seq, ATAC-seq, and ChIP-seq datasets generated in this study are available in the GEO repository with record number GSE142105. The Ingenuity Pathway Analysis (IPA) database is accessible at https://digitalinsights.qiagen.com/products-overview/discoveryinsights-portfolio/analysis-and-visualization/qiagen-ipa/. The data and any unique materials are available from the corresponding author upon reasonable request. Source data are provided with this paper.

Received: 17 March 2020; Accepted: 17 June 2021; Published online: 16 July 2021

\section{References}

1. Heitz, E. Das Heterochromatin der Moose. Jahr.ücher f.ür. Wissenschaftliche Botanik 69, 762-818 (1928).

2. Allshire, R. C. \& Madhani, H. D. Ten principles of heterochromatin formation and function. Nat. Rev. Mol. Cell Biol. 19, 229-244 (2018).

3. Janssen, A., Colmenares, S. U. \& Karpen, G. H. Heterochromatin: guardian of the genome. Annu. Rev. Cell Dev. Biol. 34, 265-288 (2018).

4. Rea, S. et al. Regulation of chromatin structure by site-specific histone $\mathrm{H} 3$ methyltransferases. Nature 406, 593-599 (2000).

5. Peters, A. H. F. M. et al. Partitioning and plasticity of repressive histone methylation states in mammalian chromatin. Mol. Cell 12, 1577-1589 (2003).

6. Matsui, T. et al. Proviral silencing in embryonic stem cells requires the histone methyltransferase ESET. Nature 464, 927-931 (2010).

7. Karimi, M. M. et al. DNA methylation and SETDB1/H3K9me3 regulate predominantly distinct sets of genes, retroelements, and chimeric transcripts in mESCs. Cell Stem Cell 8, 676-687 (2011).

8. Tachibana, M. et al. G9a histone methyltransferase plays a dominant role in euchromatic histone H3 lysine 9 methylation and is essential for early embryogenesis. Genes Dev. 16, 1779-1791 (2002).

9. Tachibana, M. et al. Histone methyltransferases G9a and GLP form heteromeric complexes and are both crucial for methylation of euchromatin at H3-K9. Genes Dev. 19, 815-826 (2005).

10. Bannister, A. J. et al. Selective recognition of methylated lysine 9 on histone H3 by the HP1 chromo domain. Nature 410, 120-124 (2001).

11. Lachner, M., O'Carroll, D., Rea, S., Mechtler, K. \& Jenuwein, T. Methylation of histone H3 lysine 9 creates a binding site for HP1 proteins. Nature 410 , 116-120 (2001).

12. Fritsch, L. et al. A subset of the histone H3 lysine 9 methyltransferases Suv39h1, G9a, GLP, and SETDB1 participate in a multimeric complex. Mol. Cell 37, 46-56 (2010)

13. Pinheiro, I. et al. Prdm 3 and Prdm16 are H3K9me1 methyltransferases required for mammalian heterochromatin integrity. Cell 150, 948-960 (2012). 
14. Towbin, B. D. et al. Step-wise methylation of histone H3K9 positions heterochromatin at the nuclear periphery. Cell 150, 934-947 (2012).

15. Zeller, P. et al. Histone H3K9 methylation is dispensable for Caenorhabditis elegans development but suppresses RNA:DNA hybrid-associated repeat instability. Nat. Genet. 48, 1385-1395 (2016).

16. Padeken, J. et al. Synergistic lethality between BRCA1 and H3K9me2 loss reflects satellite derepression. Genes Dev. 33, 436-451 (2019).

17. Rogakou, E. P., Pilch, D. R., Orr, A. H., Ivanova, V. S. \& Bonner, W. M. DNA double-stranded breaks induce histone H2AX phosphorylation on serine 139 . J. Biol. Chem. 273, 5858-5868 (1998).

18. Lemaitre, C. \& Soutoglou, E. Double strand break (DSB) repair in heterochromatin and heterochromatin proteins in DSB repair. DNA Repair 19, 163-168 (2014).

19. Crasta, K. et al. DNA breaks and chromosome pulverization from errors in mitosis. Nature 482, 53-58 (2012).

20. Tsumura, A. et al. Maintenance of self-renewal ability of mouse embryonic stem cells in the absence of DNA methyltransferases Dnmt1, Dnmt3a and Dnmt3b. Genes Cells 11, 805-814 (2006).

21. Kato, M., Takemoto, K. \& Shinkai, Y. A somatic role for the histone methyltransferase Setdb1 in endogenous retrovirus silencing. Nat. Commun. 9, 1683 (2018).

22. Martens, J. H. A. et al. The profile of repeat-associated histone lysine methylation states in the mouse epigenome. EMBO J. 24, 800-812 (2005).

23. Roulois, D. et al. DNA-demethylating agents target colorectal cancer cells by inducing viral mimicry by endogenous transcripts. Cell 162, 961-973 (2015).

24. Chiappinelli, K. B. et al. Inhibiting DNA methylation causes an interferon response in cancer via dsRNA including endogenous retroviruses. Cell 162, 974-986 (2015).

25. Colombo, D. F., Burger, L., Baubec, T. \& Schübeler, D. Binding of high mobility group A proteins to the mammalian genome occurs as a function of AT-content. PLoS Genet. 13, e1007102 (2017).

26. Jagannathan, M., Cummings, R. \& Yamashita, Y. M. A conserved function for pericentromeric satellite DNA. eLife 7, e34122 (2018).

27. Mizuguchi, T., Barrowman, J. \& Grewal, S. I. S. Chromosome domain architecture and dynamic organization of the fission yeast genome. FEBS Lett. 589, 2975-2986 (2015)

28. Towbin, B. D., Gonzalez-Sandoval, A. \& Gasser, S. M. Mechanisms of heterochromatin subnuclear localization. Trends Biochemical Sci. 38, 356-363 (2013).

29. Saksouk, N. et al. Redundant mechanisms to form silent chromatin at pericentromeric regions rely on BEND3 and DNA methylation. Mol. Cell 56, 580-594 (2014).

30. Puschendorf, M. et al. PRC1 and Suv39h specify parental asymmetry at constitutive heterochromatin in early mouse embryos. Nat. Genet. 40 , 411-420 (2008).

31. Du, J., Johnson, L. M., Jacobsen, S. E. \& Patel, D. J. DNA methylation pathways and their crosstalk with histone methylation. Nat. Rev. Mol. Cell Biol. 16, 519-532 (2015).

32. Bulut-Karslioglu, A. et al. Suv39h-dependent H3K9me3 marks intact retrotransposons and silences LINE elements in mouse embryonic stem cells. Mol. Cell 55, 277-290 (2014).

33. Liu, X. et al. UHRF1 targets DNMT1 for DNA methylation through cooperative binding of hemi-methylated DNA and methylated H3K9. Nat. Commun. 4, 1563 (2013).

34. Zhao, Q. et al. Dissecting the precise role of H3K9 methylation in crosstalk with DNA maintenance methylation in mammals. Nat. Commun. 7, 12464 (2016).

35. Baubec, T. et al. Genomic profiling of DNA methyltransferases reveals a role for DNMT3B in genic methylation. Nature 520, 243-247 (2015).

36. Kim, J. et al. Maternal Setdb1 is required for meiotic progression and preimplantation development in mouse. PLoS Genet 12, e1005970 (2016).

37. Južnić, L. et al. SETDB1 is required for intestinal epithelial differentiation and the prevention of intestinal inflammation. Gut 70, 485-498 (2021).

38. Falnes, P. Ø., Jakobsson, M. E., Davydova, E., Ho, A. \& Małecki, J. Protein lysine methylation by seven- $\beta$-strand methyltransferases. Biochem. J. 473, 1995-2009 (2016).

39. Pace, L. et al. The epigenetic control of stemness in CD8+ T cell fate commitment. Science 359, 177-186 (2018).

40. Nicetto, D. et al. H3K9me3-heterochromatin loss at protein-coding genes enables developmental lineage specification. Science 363, 294-297 (2019).

41. Larson, A. G. et al. Liquid droplet formation by HP1 $\alpha$ suggests a role for phase separation in heterochromatin. Nature 547, 236-240 (2017).

42. Strom, A. R. et al. Phase separation drives heterochromatin domain formation. Nature 547, 241-245 (2017).

43. Solovei, I. et al. LBR and Lamin A/C sequentially tether peripheral heterochromatin and inversely regulate differentiation. Cell 152, 584-598 (2013).

44. Gonzalez-Sandoval, A. et al. Perinuclear anchoring of H3K9-methylated chromatin stabilizes induced cell fate in C. elegans embryos. Cell 163 1333-1347 (2015).
45. Ran, F. A. et al. Genome engineering using the CRISPR-Cas9 system. Nat. Protoc. 8, 2281-2308 (2013).

46. Shechter, D., Dormann, H. L., Allis, C. D. \& Hake, S. B. Extraction, purification and analysis of histones. Nat. Protoc. 2, 1445-1457 (2007).

47. Meert, P. et al. Tackling aspecific side reactions during histone propionylation: the promise of reversing overpropionylation. Proteomics 16, 1970-1974 (2016).

48. Rappsilber, J., Mann, M. \& Ishihama, Y. Protocol for micro-purification, enrichment, pre-fractionation and storage of peptides for proteomics using StageTips. Nat. Protoc. 2, 1896-1906 (2007).

49. Musa, Y. R., Boller, S., Puchalska, M., Grosschedl, R. \& Mittler, G. Comprehensive proteomic investigation of Ebfl heterozygosity in Pro-B lymphocytes utilizing data independent acquisition. J. Proteome Res. 17, 76-85 (2018).

50. Kelstrup, C. D., Young, C., Lavallee, R., Nielsen, M. L. \& Olsen, J. V. Optimized fast and sensitive acquisition methods for shotgun proteomics on a quadrupole orbitrap mass spectrometer. J. Proteome Res. 11, 3487-3497 (2012).

51. Ficz, G. et al. Dynamic regulation of 5-hydroxymethylcytosine in mouse ES cells and during differentiation. Nature 473, 398-402 (2011).

52. Martin, M. Cutadapt removes adapter sequences from high-throughput sequencing reads. EMBnet. J. 17, 10-12 (2011).

53. Dobin, A. et al. STAR: ultrafast universal RNA-seq aligner. Bioinformatics 29, 15-21 (2013).

54. Jin, Y., Tam, O. H., Paniagua, E. \& Hammell, M. TEtranscripts: a package for including transposable elements in differential expression analysis of RNA-seq datasets. Bioinformatics 31, 3593-3599 (2015)

55. Love, M. I., Huber, W. \& Anders, S. Moderated estimation of fold change and dispersion for RNA-seq data with DESeq2. Genome Biol. 15, 550 (2014).

56. Ramírez, F. et al. deepTools2: a next generation web server for deepsequencing data analysis. Nucleic Acids Res. 44, W160-W165 (2016)

57. Bao, W., Kojima, K. K. \& Kohany, O. Repbase Update, a database of repetitive elements in eukaryotic genomes. Mob. DNA 6, 11 (2015).

58. Li, H. \& Durbin, R. Fast and accurate long-read alignment with BurrowsWheeler transform. Bioinformatics 26, 589-595 (2010).

59. Robinson, J. T. et al. Integrative genomics viewer. Nat. Biotechnol. 29, 24-26 (2011).

60. Buenrostro, J. D., Wu, B., Chang, H. Y. \& Greenleaf, W. J. ATAC-seq: a method for assaying chromatin accessibility genome-wide. Curr. Protoc. Mol. Biol. 109, 21.29.1-9 (2015).

61. Chen, S., Zhou, Y., Chen, Y. \& Gu, J. fastp: an ultra-fast all-in-one FASTQ preprocessor. Bioinformatics 34, i884-i890 (2018).

62. Heinig, M. et al. histoneHMM: differential analysis of histone modifications with broad genomic footprints. BMC Bioinforma. 16, 60 (2015).

63. Yu, G., Wang, L.-G. \& He, Q.-Y. ChIPseeker: an R/Bioconductor package for ChIP peak annotation, comparison and visualization. Bioinformatics 31, 2382-2383 (2015)

64. Bhardwaj, V. et al. snakePipes: facilitating flexible, scalable and integrative epigenomic analysis. Bioinformatics 35, 4757-4759 (2019).

65. Wickham, H. Elegant graphics for data analysis (ggplot2). Appl. Spatial Data Anal. R. (2009)

66. Deerinck, T. J. et al. Enhancing serial block-face scanning electron microscopy to enable high resolution 3-D nanohistology of cells and tissues. Microscopy Microanalysis 16, 1138-1139 (2010).

\section{Acknowledgements}

We are grateful to Yoichi Shinkai (RIKEN Saitama, Japan) for sharing Eset1-conditional MEF cells. We thank Jörg Büscher and the Metabolomics facility, Ulrike Bönisch, Stephanie Falk and the Deep Sequencing facility and Konrad Schuldes, Sebastian Hobitz and the Flow Cytometry Facility at the MPI-IE for processing samples; Thomas Clapes, Aikaterini Polyzou, and Eirini Trompouki for their input on ATAC-seq protocol and analysis; Roland Pohlmeyer and Sergiy Avilov from the Imaging facility at the MPI-IE for help with image acquisition and analysis. We also thank the members of the Jenuwein laboratory for insightful discussions and for sharing reagents and protocols. Research in the laboratory of T.J. is supported by the Max Planck Society and by additional funds from the German Research Foundation (DFG) within the CRC992 consortium "MEDEP". Research in the laboratory of C.G. was funded by the Friedrich Miescher Institute for Biomedical Research, which is supported by the Novartis Research Foundation.

\section{Author contributions}

Conceptualization, T.M. and T.J.; methodology, T.M.; investigation, T.M., N.S., Y.M., G. M., B.E., A.G.M., and C.G.; formal analysis, M.O.-S., D.R., Y.M., and G.E.; writingoriginal draft, T.M. and T.J.; supervision, T.J.

\section{Funding}

Open Access funding enabled and organized by Projekt DEAL.

\section{Competing interests}

The authors declare no competing interests. 


\section{Additional information}

Supplementary information The online version contains supplementary material available at https://doi.org/10.1038/s41467-021-24532-8.

Correspondence and requests for materials should be addressed to T.J.

Peer review information Nature Communications thanks the anonymous reviewers for their contribution to the peer review of this work.

Reprints and permission information is available at http://www.nature.com/reprints

Publisher's note Springer Nature remains neutral with regard to jurisdictional claims in published maps and institutional affiliations. (c) (i) Open Access This article is licensed under a Creative Commons Attribution 4.0 International License, which permits use, sharing, adaptation, distribution and reproduction in any medium or format, as long as you give appropriate credit to the original author(s) and the source, provide a link to the Creative Commons license, and indicate if changes were made. The images or other third party material in this article are included in the article's Creative Commons license, unless indicated otherwise in a credit line to the material. If material is not included in the article's Creative Commons license and your intended use is not permitted by statutory regulation or exceeds the permitted use, you will need to obtain permission directly from the copyright holder. To view a copy of this license, visit http://creativecommons.org/licenses/by/4.0/.

(C) The Author(s) 2021 